\title{
Complex aero-engine intake ducts and dynamic distortion
}

\author{
David G. MacManus ${ }^{1}$, Nicola Chiereghin ${ }^{2}$, Daniel Gil Prieto ${ }^{3}$ and Pavlos Zachos ${ }^{4}$ \\ Cranfield University, Bedfordshire, MK43 OAL, United Kingdom
}

For many embedded engine systems, the intake duct geometry introduces flow distortion and unsteadiness which must be understood when designing the turbomachinery components. The aim of this work is to investigate the capabilities of modern computational methods for these types of complex flows, to study the unsteady characteristics of the flow field and to explore the use of proper orthogonal decomposition methods to understand the nature of the unsteady flow distortion. The unsteady flows for a range of S-duct configurations have been simulated using a delayed detached eddy simulation method. Analysis of the conventional distortion criteria highlights the main sensitivities to the S-duct configuration and quantifies the unsteady range of these parameters. The unsteady flow field shows signature regions of unsteadiness which are postulated to be related to the classical secondary flows as well as to the streamwise flow separation. A proper orthogonal decomposition of the total pressure field at the duct exit identifies the underpinning flow modes which are associated with the overall total pressure unsteadiness distributions. Overall, the unsteady distortion metrics are not found to be solely linked to a particular proper orthogonal decomposition mode, but are dependent on a wider range of modes.

\section{Nomenclature}

$\begin{array}{ll}p & =\text { static pressure } \\ p_{o} & =\text { total pressure } \\ r & =\text { cross section radius } \\ \text { peak } & =\text { maximum value of a temporal distribution } \\ \max & =\text { maximum value of a spatial distribution }\end{array}$

\footnotetext{
${ }^{1}$ Senior Lecturer, Propulsion Engineering Centre, Building 52, Cranfield University, AIAA Member.

${ }^{2} \mathrm{PhD}$ Student, Propulsion Engineering Centre, Building 52, Cranfield University.

${ }^{3}$ PhD Student, Propulsion Engineering Centre, Building 52, Cranfield University.

${ }^{4}$ Lecturer, Propulsion Engineering Centre, Building 52, Cranfield University, AIAA Member.
} 


$$
\begin{aligned}
& \min =\text { minimum value of a spatial distribution } \\
& t_{c} \quad=\text { convective time }=L_{s} / w_{i n} \\
& u, v, w=\text { velocity vector components } \\
& v_{\theta} \quad=\text { tangential velocity } \\
& x, y, z=\text { system of reference coordinates } \\
& D \quad=\text { diameter } \\
& \mathrm{H}_{0} \quad=\quad \text { Duct vertical offset } \\
& L_{s} \quad=\quad \mathrm{S} \text {-Duct length measured along the centreline } \\
& M \quad=\text { Mach number } \\
& \mathrm{Q}=\mathrm{Q} \text {-criterion, } \frac{1}{2}\left(\Omega^{2}-S^{2}\right) \\
& R=\mathrm{S} \text {-Duct centreline curvature radius } \\
& \text { Re }=\text { diameter-based Reynolds number } \\
& \mathrm{S} \quad=\text { strain rate } \\
& \text { St } \quad=\text { Strouhal number } \\
& \alpha \quad=\text { swirl angle } \\
& \Delta t \quad=\text { unsteady simulation time step } \\
& \Delta t^{*}=\text { non-dimensional time step, } \Delta \mathrm{t} / \mathrm{t}_{\mathrm{c}} \\
& \Delta z_{\text {out }}=\text { distance downstream the S-Duct outlet along the z-axis } \\
& \Theta \quad=\text { angular position on the AIP } \\
& \Phi \quad=\text { S-Duct angular position } \\
& \Omega \quad=\text { vorticity }
\end{aligned}
$$

$$
\begin{array}{ll}
\text { Subscripts } & \\
\text { AIP } & =\text { aerodynamic interface plane } \\
\text { Avg } & =\text { area-average } \\
\text { in } & =\text { inlet } \\
\mathrm{x}, \mathrm{y}, \mathrm{z} & =\text { Cartesian co-ordinate directions } \\
\Theta \quad & \text { tangential direction }
\end{array}
$$




$$
\begin{aligned}
& <p 0>\quad=\text { referred to the time-averaged distribution of } \mathrm{p} 0 \\
& <\alpha>\quad=\text { referred to the time-averaged distribution of } \alpha
\end{aligned}
$$

\section{Operators}

$$
\begin{array}{ll}
\langle\cdot\rangle & =\text { time-average } \\
- & =\text { area-average } \\
\sigma \quad & =\text { standard deviation } \\
i & =\text { ring index where } \mathrm{i}=0 \text { refers to the inner ring }
\end{array}
$$

\section{Introduction}

Future aircraft engine configurations are expected to require a closer coupling between the propulsion system and the airframe, with potential benefits in term of drag, noise and overall performance. For many embedded and partially-embedded engine systems, the complexity of the flow field associated with convoluted intakes presents an area of notable research challenges. The development of embedded engine configurations leads to the intake and duct aerodynamics having greater importance since they play a vital role in engine operability and performance ${ }^{1}$. The convolution of the intake duct introduces additional flow distortion and unsteadiness which must be understood and quantified when designing the turbomachinery components. Current industry practice for characterising distorted flow fields into compressor or fan systems is known to have limitations for predicting instability onset ${ }^{1,2}$.

Complex intake aerodynamic distortion can be characterized by total pressure, temperature and swirl non uniformities. A loss of performance in term of surge margin is observed when the distorted area extends circumferentially beyond a critical angle ${ }^{1}$ which depends on the compressor characteristics. Several metrics have been proposed to quantify total pressure distortion from measurements ${ }^{3}$. Swirl can be related to vortices and secondary flow and modifies the nominal incidence angle of the flow to the blade with the risk of unexpected stall. A further degradation of engine performance was observed when total pressure distortion is combined with a bulk swirl counter-rotating with respect to the compressor rotation direction ${ }^{2}$. This was also evident in the experimental investigation of vortex ingestion by Mitchell ${ }^{4}$, with the greatest effect observed when a counter-rotating vortex was located close to the compressor hub ${ }^{5}$. The effect of a twin swirl pattern was experimentally observed in Mayer et $\mathrm{al}^{6}$, where twin vortices were generated by means of a delta wing with variable angle of attack where an increase in the average vortex swirl angle was observed to produce an increase in loss of surge margin. A reduction of $26 \%$ of the 
surge margin was observed with an average swirl angle of 13 degrees, together with a reduction of $7 \%$ of the compressor efficiency.

Previous studies noted that engine stall can be generated from distortion fluctuations ${ }^{7}$ and highlighted the importance of the unsteady aspects. Further studies of dynamic distortion emphasized the importance of instantaneous distortion and in particular the local peak values ${ }^{7}$. Within this context, the aim of the current work is to investigate the capabilities of modern computational methods for these types of complex flows, to study the unsteady characteristics of the flow field and to explore the use of proper orthogonal decomposition methods to understand the nature of the unsteady flow distortion.

A number of studies have assessed the flow distortion associated with S-duct configurations. Wellborn et $\mathrm{al}^{8}$ undertook an experimental study of the flow through a diffusing S-duct. The total pressure distribution at the AIP showed a low-pressure zone on the lower sector associated with a large separation generated in the first part of the duct. The flow field also featured strong secondary flows which led to the generation of a pair of contra-rotating vortices at the AIP. This test case has been used to evaluate computational methods and a comparison between different turbulence models for steady Reynolds Averaged Navier Stokes (RANS) simulations was performed by Delot et $\mathrm{al}^{9}$ and Fiola et. $\mathrm{al}^{10}$. These results show a reasonable capability of well-established turbulence models, like Spalart-Allmaras and k- $\omega$ SST, to represent the behaviour of highly separated flow typical of a highly convoluted intake. Although the models could not accurately predict all the performance parameters such as pressure recovery and total pressure distortion, it demonstrated the capability of k- $\omega$ SST to broadly capture the flow physics. However, high bandwidth pressure measurements in two S-duct configurations with different centreline offsets ${ }^{11,12,13}$ demonstrated notable unsteadiness in the separation region which produced oscillations of the flow distortions at the duct exit plane. The measurement of the total pressure standard deviation at the AIP reported in Delot et al $^{12}$ outlined fluctuations up to $3.5 \%$ of the intake total pressure with an AIP Mach number of about 0.4 .

Large Eddy Simulations (LES) directly resolve the turbulent structures which are larger than the numerical grid and use a universal model to simulate the effect of sub-grid scale. These methods require substantial resources and a compromise is offered by Detached-Eddy simulations (DES) where the RANS equations are applied close to the wall, while the rest of the flow is solved by LES equations. Berens et $\mathrm{al}^{14}$ and $\operatorname{Delot}^{15}$ demonstrated the capability of DES to reproduce the strongly turbulent behaviour of the flow observed in curved intakes. Both these studies compared steady and unsteady RANS methods with Zonal-DES ${ }^{16}$ and DES combined with Spalart-Allmaras and 
algebraic Reynolds stress turbulence models. These studies demonstrated the capability of DES to capture the highly unsteady nature of flow separations in S-Ducts ${ }^{14}$ and serpentine ducts ${ }^{15}$.

This current research extends the work on flow distortion simulation with a study of the effect of duct vertical offset, Reynolds number and Mach number. The capability of DES to predict the change in performance for different configurations is assessed through a comparison with experimental data ${ }^{12,13}$. For a better understanding of the dynamic distortion characteristics, a Proper Orthogonal Decomposition (POD) of the total pressure variations at the S-Duct exit plane is also performed.

\section{S-duct configurations}

In this research, the S-Duct configurations previously experimentally investigated by Wellborn ${ }^{8}$, Garnier $^{13}$ and Delot $^{12}$ were considered (Fig. 1). A diffusion area ratio of 1.52 was fixed for all the configurations (Table 1). In this work, three different geometric configurations were considered with the main parameters defined in Table 1. S-Duct 1 has the same dimensions as the geometry experimentally investigated by Wellborn et $\mathrm{a}^{8}$. The centreline curvature distribution is defined using two circular arcs with the same radius of curvature and angular extent. The circular cross sections are normal to the centreline and the area distribution follows as cubic function as described by Wellborn ${ }^{8}$. S-Duct 2 maintains the same non-dimensional offset $H_{0} / L$ and length $L / D_{\text {in }}$ as in $S-D u c t ~ 1$ but with a reduced inlet diameter and concomitant reduction in Reynolds number (Table 2). S-Duct 3 is related to S-Duct 2 but with an increase of the offset $\mathrm{H}_{0} / \mathrm{L}$ from 0.268 to 0.493 . These configurations were used to perform a range of simulations with required massflow for the Mach number at the AIP (Table 2). The two flow conditions investigated in this work correspond to the experimental cases studied by Delot et al. ${ }^{9}$, which are $\mathrm{M}_{\mathrm{AIP}}=0.18$ and 0.36 . The comparison between S-Duct 1 and S-Duct 2A provides an assessment of the effect of the geometric scaling on the SDuct performance. An assessment of the influence of Mach number is obtained from S-Duct 2A and 2B as well as S-Duct 3A and S-Duct 3B. Finally, the assessment of the effect of the offset $\left(\mathrm{H}_{0} / \mathrm{L}\right)$ is obtained from the comparison between S-Duct 2 and 3. These assessments are performed on an aerodynamic interface plane (AIP) at a distance $\Delta \mathrm{z}_{\text {out }}$ of $0.5 \mathrm{D}_{\text {in }}$ downstream the S-Duct outlet (Fig. 1). For both S-Duct 2 and 3 the computational domain included a constant area cylindrical section with a length of $4 \mathrm{D}_{\text {in }}$ upstream of the inlet plane of the S-Duct.

For validation, two separate simulations have been performed with the geometry in Fig. 1b, for high Mach number, S-Duct 2AS, and low Mach number, S-Duct 2BS, which model the experimental configuration with a spinner downstream of the S-duct ${ }^{9,12}$. Downstream of the spinner extends a cylindrical section with constant area 
and parallel walls. A static pressure boundary condition was applied at the duct exit and adjusted to provide the requirement massflow. The inlet total pressure profile from the experiment ${ }^{9}$ was applied at the inlet to the CFD domain which was $0.57 \mathrm{D}_{\text {in }}$ upstream of the start of the duct. The comparison between numerical and experimental results is performed at the $\mathrm{AIP}_{\text {exp }}$ section (Fig. 1b), at $\Delta \mathrm{z}_{\text {out }}$ of $0.36 \mathrm{D}_{\text {in }}$ and ahead of the spinner section, where unsteady total pressure measurements with 40 high-bandwidth transducers were taken by Delot et $\mathrm{al}^{12}$. The experimental model was manufactured and tested at the ONERA wind tunnel R4MA (Modane-Avrieux, France) in 2006 and 2008. The geometry is based on a smaller scale model developed by Harloff et al. at NASA Lewis Research Center in the $1990 \mathrm{~s}^{0}$.

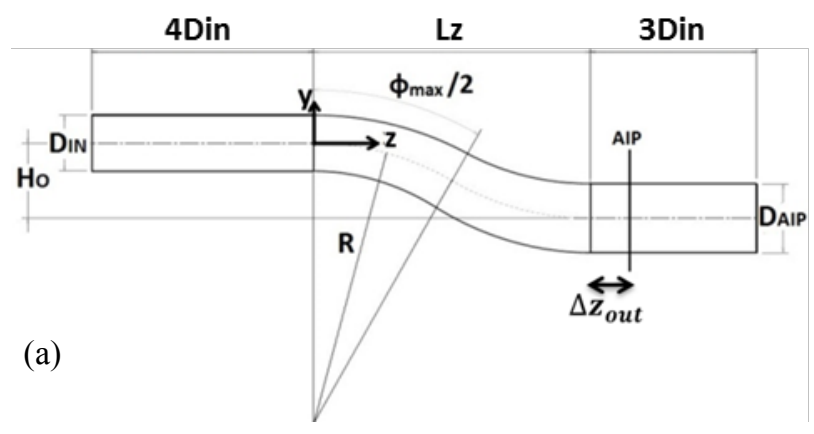

(b)

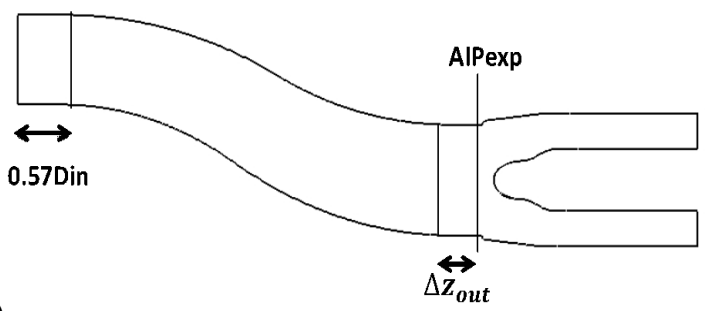

Fig. 1 (a) Schematic of S-duct (b) geometrical representation of the S-Duct 2S from Delot et al. ${ }^{12,13}$ 
Table 1 S-duct geometry parameters

\begin{tabular}{cccc}
\hline \hline Parameters & S-Duct 1 & S-Duct 2 & S-Duct 3 \\
\hline $\mathrm{A}_{\text {out }} / \mathrm{A}_{\text {in }}$ & 1.52 & 1.52 & 1.52 \\
$\mathrm{~L} / \mathrm{D}_{\text {in }}$ & 5.0 & 5.0 & 4.95 \\
$\mathrm{H}_{\mathrm{o}} / \mathrm{L}$ & 0.268 & 0.268 & 0.493 \\
$\phi_{\max }\left[{ }^{\circ}\right]$ & 60 & 60 & 105.1 \\
$\mathrm{D}_{\text {in }}[\mathrm{m}]$ & 0.2042 & 0.133 & 0.133 \\
$\mathrm{D}_{\text {out }}[\mathrm{m}]$ & 0.2514 & 0.164 & 0.164 \\
$\mathrm{R}[\mathrm{m}]$ & 1.021 & 0.665 & 0.4146 \\
$\mathrm{~L}[\mathrm{~m}]$ & 1.021 & 0.665 & 0.658 \\
$\mathrm{H}_{\mathrm{o}}[\mathrm{m}]$ & 0.2736 & 0.1784 & 0.3245 \\
\hline
\end{tabular}

Table 2 : Test cases and flow conditions

\begin{tabular}{lccc}
\hline \hline \multicolumn{1}{c}{ Case } & $\mathbf{M}_{\text {AIP }}$ & Inlet Re $_{\text {Din }}$ & $\Delta \mathbf{t}$ \\
\hline S-Duct 1 & 0.36 & $2.6 \times 10^{6}$ & $6.0 \times 10^{-6} \mathrm{~s}$ \\
S-Duct 2A & 0.36 & $1.7 \times 10^{6}$ & $6.0 \times 10^{-6} \mathrm{~s}$ \\
$\begin{array}{l}\text { S-Duct 2AS } \\
\text { (with spinner) }\end{array}$ & 0.36 & $1.7 \times 10^{6}$ & $6.0 \times 10^{-6} \mathrm{~s}$ \\
& & & \\
S-Duct 2B & 0.18 & $1.1 \times 10^{6}$ & $12 \times 10^{-6} \mathrm{~s}$ \\
S-Duct 2BS & 0.18 & $1.1 \times 10^{6}$ & $12 \times 10^{-6} \mathrm{~s}$ \\
(with spinner) & & & \\
S-Duct 3A & 0.39 & $1.8 \times 10^{6}$ & $6.0 \times 10^{-6} \mathrm{~s}$ \\
S-Duct 3B & 0.18 & $1.1 \times 10^{6}$ & $12 \times 10^{-6} \mathrm{~s}$ \\
\hline
\end{tabular}

\section{Methodology}

\section{A. Distortion metrics}

This paper addresses both total pressure and swirl distortion. The importance of the effect of flow nonuniformities on an aero-engine has resulted in the development of a range of reduced order parameters to quantify the level of distortion being presented to the engine ${ }^{2,3}$. Distortion measurements are typically performed using rakes 
with 8 circumferential and 5 radial probes ${ }^{3}$. The probes are usually equi-spaced circumferentially and with a radial spacing to provide an equal area distribution ${ }^{18}$. For the computational work the spatial resolution at the AIP is several orders of magnitude greater than that of an $8 \times 5$ rake. The aerodynamic parameters are linearly interpolated from the computational mesh onto the $8 \times 5$ loci. The area-average total pressure of the AIP, $\left(\overline{p 0_{A I P}}\right)$, is calculated as the mean value of these 40 points.

\section{Total pressure distortion descriptors}

The total pressure distortion at the AIP is usually quantified in terms of sectional distortion coefficient (DC60), circumferential distortion index (CDI) and radial distortion index (RDI). DC60 is defined by the difference between the average total pressure $\left(\overline{p 0_{A I P}}\right)$ and the lowest average total pressure in a sector of $60^{\circ}$ angle $\left(\overline{p 0_{60}}\right)$ and is nondimensionalized by the mean dynamic head of the whole $\operatorname{AIP}^{19}$ (Eq. 1):

$$
D C(60)=\frac{\overline{p 0_{A I P}}-\overline{p 0_{60}}}{\overline{q_{A I P}}}
$$

The circumferential distortion index (CDI) assesses the uniformity of the circumferential total pressure distribution at a specific radial position and is defined in Eq. $2^{3}$ :

$$
C D I=\operatorname{Max}_{i=0}^{3}\left(0.5\left[\frac{\overline{p 0}_{i}-\min (p 0)_{i}}{\overline{p 0_{A I P}}}+\frac{\overline{p 0}_{i+1}-\min (p 0)_{i+1}}{\overline{p 0_{A I P}}}\right]\right)
$$

where $\overline{p 0_{A I P}}$ is the average total pressure at the AIP, $\overline{p 0}_{\mathrm{i}}$ is the average total pressure of the circumferential pressure distribution of the $\mathrm{i}$-th ring and $\min (p 0)_{i}$ is the minimum pressure along this same ring. Non-uniformities in the radial distortion were evaluated by the radial distortion index (RDI) which is defined in Eq. $3^{3}$ :

$$
R D I=\operatorname{Max}\left(\frac{\overline{p 0_{A I P}}-\overline{p 0_{i=0}}}{\overline{p_{A I P}}}, \frac{\overline{p_{A I P}}-\overline{p 0_{i=4}}}{\overline{p 0_{A I P}}}\right)
$$

with $\overline{p 0}_{i=0}$ the average total pressure of the pressure distribution of the inner ring and $\overline{p 0}_{i=4}$ is the average total pressure at the outer ring.

Additional metrics have been developed ${ }^{18}$ which consider total pressure distortion as a ring-based radial distribution of specific parameters where radial and circumferential distortions are quantified separately. The circumferential distortion is defined in term of extent, intensity and multiple-per-revolution parameters. The circumferential total pressure distribution $\mathrm{p} 0$ is considered in terms of an average $\overline{p 0}_{i}$ and regions of low relative total pressure $\left(\mathrm{p} 0<\overline{p 0}_{i}\right)$ and high relative total pressure $\left(\mathrm{p} 0>\overline{p 0}_{i}\right)$. The extent element, $\theta_{i}^{-}$, is defined as the 
circumferential extension of the low pressure region ${ }^{18}$. If only a single low pressure region is obtained for an individual circumferential ring $i$, the extent parameter is calculated as follows:

$$
\theta_{i}^{-}=\theta 2_{i}-\theta 1_{i}
$$

The extent parameter provides an indication of the time spent in the low pressure zone by a compressor blade during a revolution period. Significant effects on performance are observed only for $\theta_{i}^{-}$higher than a critical angle depending on the compressor time constant and rotation $\operatorname{speed~}^{20}$. Another aspect of the circumferential distortion to be considered is its amplitude, which is quantified by the circumferential distortion intensity for the ring $\mathrm{i}$ is defined as:

$$
\left(\Delta P_{C} / P\right)_{i}=\left(\frac{{\overline{p 0_{i}}}_{i} \overline{p 0_{-}}}{\overline{p 0}_{i}}\right)_{i}
$$

where:

$$
\left(\overline{p 0_{-}}\right)_{i}=\frac{1}{\theta_{i}^{-}} \int_{\theta 1_{i}}^{\theta 2_{i}} p 0(\theta)_{i} d \theta
$$

In the case of multiple pressure distortion regions, a circumferential distortion intensity $\left(\Delta P_{C} / P\right)_{i, k}$ and extent $\theta_{i, k}^{-}$are calculated for every low pressure region $\mathrm{k}$, and the extent and circumferential intensity value assigned to the ring $\mathrm{i}$ is the one corresponding to the low pressure region with the biggest area, that is ${ }^{18}$ :

$$
\begin{aligned}
\left(\Delta P_{C} / P\right)_{i} & =\left(\Delta P_{C} / P\right)_{i, k \max } \\
\theta_{i}^{-} & =\theta_{i, k \max }^{-}
\end{aligned}
$$

where:

$$
(k \max )_{i}=\left(\underset{k=1, . ., Q}{\operatorname{argmax}}\left(\Delta P_{C} / P\right)_{i, k} \theta_{i, k}^{-}\right)_{i}
$$

and $\mathrm{Q}$ is the number of low pressure region. The circumferential extension of this region is the value assigned to the extent element $\theta_{i}^{-}$of the ring. A pattern of two or more adjacent low pressure regions separated by high pressure regions with extension smaller than a reference circumferential extension $\theta_{\min }^{+}$is considered as a single equivalent low pressure region. In this work it is taken as $25^{\circ}$. The extent assigned to such equivalent region is the sum of the extent of the low pressure regions which compound the pattern: 


$$
\theta_{i, k}^{-}=\sum_{k p=1}^{Q P} \theta_{i, k p}^{-}
$$

where QP is the number of low pressure region in the pattern. Its equivalent circumferential intensity is defined as the extent-weighted value of the component regions:

$$
\left(\Delta P_{C} / P\right)_{i, k}=\frac{\sum_{k p=1}^{Q P}\left[\left(\Delta P_{C} / P\right)_{i, k p} \theta_{i, k p}^{-}\right]}{\sum_{k p}^{Q P} \theta_{i, k p}^{-}}
$$

The radial total pressure distortion is calculated from the difference between the average total pressure of the ring $\mathrm{i}$ $\overline{p 0}_{i}$ and the average of the whole AIP $\left(\overline{p 0_{A I P}}\right)$ :

$$
\left(\Delta P_{R} / P\right)_{i}=\frac{\overline{p 0_{A I P}}-\overline{p 0}_{i}}{\overline{p 0_{A I P}}}
$$

The SAE descriptors also provide a radial distribution of distortion parameters rather than a single value. For this reason $\max \left(\Delta P_{C} / P\right)$ and $\overline{\Delta P_{C} / P}$ are defined as:

$$
\begin{gathered}
\max \left(\Delta P_{C} / P\right)=\max _{i=0, \ldots, 4}\left(\Delta P_{C} / P\right)_{i} \\
\overline{\Delta P_{C} / P}=\frac{1}{5} \sum_{i=0}^{4}\left(\Delta P_{C} / P\right)_{i}
\end{gathered}
$$

The maximum radial distortion $\max \left(\Delta P_{R} / P\right)$ and its average value $\overline{\Delta P_{R} / P}$ are also similarly defined. The maximum value of intensity $\max \left(\Delta P_{C} / P\right)$ indicates the greatest distortion intensity applied to a blade and is a useful parameter in a first assessment of intake/engine compatibility.

\section{Swirl distortion descriptors}

The distortion descriptors for swirl based non-uniformities are considered in terms of an $\mathrm{SC}^{10}{ }^{19}$ and a swirl intensity parameter ${ }^{2}$. SC60 is defined as the ratio of $\max \left(\left|\overline{V_{\theta_{60}} \mid}\right|\right)$, the maximum average circumferential velocity in a section of $60^{\circ}$, to the mean axial velocity $\overline{w_{A I P}}$ and is a modification of the formulation used in Zantopp et $\mathrm{al}^{21}$ :

$$
S C(60)=\frac{\max \left(\mid \overline{V_{\theta_{60}}}\right)}{\overline{w_{A I P}}}
$$

Swirl intensity (SI) is a parameter which has been used to quantify swirl distortion ${ }^{2}$. The swirl angle is defined as the circumferential angle from the axial direction of the velocity vector, and is considered positive in the counterclockwise direction looking at the AIP from downstream.: 


$$
\alpha=\tan ^{-1}\left(\frac{v_{\theta}}{w}\right)
$$

The swirl angle distribution is divided into positive and negative angle regions and the swirl sector elements are defined as:

$$
\begin{aligned}
& S S_{i}^{+}=\frac{1}{\theta_{i}^{+}} \int_{\theta_{i}^{+}} \alpha(\theta)_{i} d \theta \\
& S S_{i}^{-}=\frac{1}{\theta_{i}^{-}} \int_{\theta_{i}^{-}} \alpha(\theta)_{i} d \theta
\end{aligned}
$$

The radial average, maximum and overall average swirl intensity SI is defined as:

$$
\begin{gathered}
S I_{i}=\frac{S S_{i}^{+} \theta_{i}^{+}+\left|S S_{i}^{-}\right| \theta_{i}^{-}}{360} \\
\max (S I)=\max _{i=0, \ldots, 4}(S I)_{i} \\
\overline{S I}=\frac{1}{5} \sum_{i=0}^{4}(S I)_{i}
\end{gathered}
$$

\section{B. CFD method}

The calculations are performed using a Delayed Detached-Eddy-Simulation (DES) where the Delayed version of the DES (DDES) is used to prevent grid-induced separation problems ${ }^{22,23,24}$. In this current work the k- $\omega$ SST turbulence model was chosen for the closure of the RANS equations. A pressure-based solver was selected with a segregated PISO scheme ${ }^{25,26}$. A second-order spatial interpolation scheme was used for the pressure equations and the third order MUSCL scheme for momentum, energy and turbulence. An iterative time advancement scheme with a bounded second-order temporal discretization method was used ${ }^{25,26}$. The inlet boundary condition comprised specified uniform total pressure and total temperature profile for S-Duct 1, 2 and 3. For the validation cases S-Duct 2AS and 2BS, the measured boundary layer profiles were specified at the inlet plane. The static pressure was specified at the domain exit and was adjusted to provide the required average Mach number at the AIP. The inlet domain extent of $4 \mathrm{D}_{\text {in }}$ resulted in the growth of the duct boundary layer. For S-Duct 1 , Wellborn et $\mathrm{al}^{8}$ reported a measured inlet boundary layer thickness at a location of $\mathrm{Z} / \mathrm{D}_{\mathrm{i}}$ of -0.5 upstream of the S-Duct inlet plane. At an AIP Mach number of 0.36 , the measured boundary layer had a compressible displacement thickness of $0.0146 \mathrm{r}_{\text {in }}$ and a

shape factor of 1.38. The time average computed boundary layer for the medium mesh had a boundary layer displacement thickness of $0.0176 \mathrm{r}_{\text {in }}$ and shape factor of 1.39 . 


\section{Computational grid}

A baseline structured mesh with a size of 5.9 million of nodes was created using a multi-blocking strategy. To maintain the mesh orthogonality to the wall for every configuration, four different blocks were created: the first associated with the inlet straight duct, the second and the third with the S-Duct, and the forth with the cylindrical section downstream the S-Duct. The full three-dimensional geometry was simulated and the mesh has an H-grid structure in the central part of the duct, and an O-grid structure around the wall. The robustness of the method and the simplicity of the geometry allowed a good quality of the mesh for every case, with a $2 \times 2 \times 2$ determinant greater than 0.8 . The mesh was clustered towards the wall to ensure that $y+$ was less than 1 over the full domain with an expansion ratio off the wall of 1.05 . The number of nodes in the streamwise direction was between approximately 500 and 600 nodes along the duct. The number of nodes on the AIP was approximately $6.5 \mathrm{k}, 11 \mathrm{k}$ and $19 \mathrm{k}$ for the coarse, medium and fine mesh, respectively. The expansion ratio off the wall is 1.05 .

An initial grid sensitivity study has been carried out for the S-duct 2A and three RANS calculations using the k$\omega$ SST turbulence model were performed with spatial resolution of 3.1, 5.9 and 11.2 million nodes for the coarse, medium and fine meshes, respectively (Table 3). Although it is known that solutions from methods such as DDES are dependent on the grid resolution it is still considered useful to evaluate this sensitivity ${ }^{27}$. The sensitivity of the mesh dependence was considered in terms of the total pressure ratio (PR) between the inlet and the AIP and the total pressure distortion based on the circumferential distortion index (CDI). Between the medium and fine mesh the PR increased by $0.07 \%$ and CDI reduced by $0.7 \%$. The grid convergence index for CDI was $0.12 \%$ and using the Richardson extrapolation ${ }^{28}$ the discretization error was estimated at $0.8 \%$ for CDI. The effect of the mesh resolution on the unsteady aerodynamics was evaluated for S-duct 2A with a nominal AIP Mach number of 0.36 (Table 2). Unsteady calculations were performed using the same meshes as for the RANS mesh sensitivity study. An unsteady analysis using the medium mesh was performed with a time step $\Delta \mathrm{t}$ of $6 \times 10^{-6} \mathrm{~s}$. In order to maintain a similar Courant number, a time step of $5 \times 10^{-6} \mathrm{~s}$ was used for the fine mesh, and $10 \times 10^{-6} \mathrm{~s}$ for the coarse mesh. Although the local Courant number depended on the unsteady flow characteristics, across the range of coarse, medium and fine meshes it was kept below 1.0. The data was analysed for 55 convective time scales after the initial transient from the steady RANS solution. The convective time $t_{c}$ is defined in terms of the S-Duct curvilinear length and time-averaged inlet velocity. The impact of the mesh resolution on the time-averaged PR showed a variation of time-averaged PR of $0.05 \%$ from the medium to fine mesh. The distortion metrics are more sensitive to the spatial resolution due to the 
discontinuous nature of the filters which are inherent in their definitions. Total pressure distortion is evaluated in terms of various metrics including DC60, CDI and RDI. A monotonic variation was observed for the time-averaged DC60, RDI and CDI. Between the medium and fine mesh simulations the time-averaged RDI decreased by $1.8 \%$ and DC60 and CDI increased by 8.0 and 4.6\%, respectively. DC60 is defined in terms of AIP values of areaaveraged total pressure $\left(\overline{p 0_{A I P}}\right)$, dynamic head $\left(\overline{q_{A I P}}\right)$, and the $60^{\circ}$ sector with the lowest average total pressure $\left(\overline{p 0_{60}}\right)($ Eq. 1). As the mesh resolution increases, the local regions with the greatest loss in total pressure are better

resolved and there is decrease in $\overline{p 0_{60}}$. However, the increased loss is not uniformly distributed across the AIP and the average loss in $\overline{p 0_{A I P}}$ is dominated by these local losses in the worst $60^{\circ}$ sector. Consequently there is a concomitant increase in $<$ DC60 $>$. A similar effect is noted for CDI where the better resolved local loss regions associated with the increased mesh resolution result in lower values of minimum $(p 0)_{i}$. (Eq. 2) Although there are also associated smaller changes to a $\overline{p 0_{l}}$ and $\overline{p 0_{A I P}}$, overall the changes to $(p 0)_{i}$ dominate and there is an increase in $<\mathrm{CDI}>$.The swirl distortion was evaluated in terms of swirl intensity $(\overline{S I})$ with an increase of $3.2 \%$ from the medium to fine mesh. 
The impact of the mesh refinement on the unsteady aspects was also evaluated for the unsteady data of 55 mean

Table 3 Effect of grid size on distortion metrics

\begin{tabular}{cccc}
\hline \hline & \multicolumn{3}{c}{ Grid size (millions nodes) } \\
Parameter & $\mathbf{3 . 1}$ & $\mathbf{5 . 9}$ & $\mathbf{1 1 . 2}$ \\
\hline$\langle\overline{P R}\rangle$ & 0.970 & 0.969 & 0.968 \\
$\langle C D I\rangle$ & 0.067 & 0.071 & 0.076 \\
$\langle R D I\rangle$ & 0.033 & 0.032 & 0.032 \\
$\langle D C(60)>$ & 0.43 & 0.47 & 0.51 \\
$\langle\overline{S I}\rangle$ & 0.119 & 0.118 & 0.121 \\
$\sigma_{\overline{P R}}$ & 0.0023 & 0.0025 & 0.0023 \\
$\sigma_{C D I}$ & 0.0165 & 0.0149 & 0.0133 \\
$\sigma_{D C 60}$ & 0.0137 & 0.0128 & 0.0112 \\
\hline
\end{tabular}

convective time periods. The standard deviation of PR changed monotonically as the mesh size increased and there was a $5.7 \%$ decrease between the medium and fine meshes. The sensitivity of the distortion metrics was greater. Between the medium and fine mesh, a variation of up to $13 \%$ was observed for the standard deviation of the total pressure distortion parameters RDI, DC60 and CDI, while the standard deviation of swirl intensity (SI) increased by $2.1 \%$. Although the time-averaged distortion metrics of $\angle \mathrm{DC} 60>$ and $<\mathrm{CDI}>$ increase with mesh resolution, the corresponding unsteady elements reduce. For DC60 the effect of the changes in mesh resolution have a relatively minor impact on the overall terms $\overline{p 0_{A I P}}$ and $\overline{q_{A I P}}$. The dominant effect is associated with the more local loss region reflected in the $\overline{p 0_{60}}$ term. As the mesh resolution increases the calculated time-average loss also increases, but the temporal variation is reduced and therefore the $<$ DC60 $>$ increases but the $\sigma_{\text {DC60 }}$ reduces. A similar effect is noted for $\sigma_{\mathrm{CDI}}$ where the dominate term is also the local minimum $(p 0)_{i}$ (Eq. 2). The increase in spatial resolution provides greater local loss but a reduction in temporal changes and therefore an increase in the $<$ CDI $>$ but a reduction in $\sigma_{\mathrm{DC} 60}$. The results presented in this paper were performed using a medium mesh for all configurations.

\section{Computational time steps}

A time step $\Delta \mathrm{t}$ of $6 \times 10^{-6} \mathrm{~s}$ was chosen for the high Mach number cases (S-Duct 1, 2A, 2AS, 3A) and $1.2 \times 10^{-5} \mathrm{~s}$ for the low Mach cases (S-Duct 2B, 2BS, 3B) which correspond to a non-dimensional time step $\Delta$ t of approximately 0.002 with respect to the mean overall convective time through the duct. To assess the impact of the time step, the SDuct $2 \mathrm{~A}$ case was also simulated with a time step of $12 \times 10^{-6} \mathrm{~s}$ and the time average and unsteady distortion metrics 
were compared. With regard to the time-averaged values, pressure recovery and RDI decreased by less than $0.1 \%$ with time step reduction from 12 to $6 \times 10^{-6} \mathrm{~s}$. Time-averaged swirl intensity $\left.\overline{(S I}\right)$ decreased by $0.2 \%$ while CDI and DC60 increased by 3\%. The unsteady characteristics were more sensitive to the change in time step with variations of standard deviation of CDI, RDI, DC60 and SI of up 7\% with this time step reduction. A variation of $2 \%$ was observed for the standard deviation of pressure recovery.

The DDES calculations used 20 sub-iterations per time step which typically resulted in residuals in the order of $10^{-6}$ for continuity equation and $10^{-7}$ for momentum, energy, $\mathrm{k}$ and $\omega$ equations, with a reduction of at least three orders of magnitude of all the residuals for each time step. For all the cases, a discarded interval of 15 convective times where used as a transition between the steady solution and the established unsteady flow field. To assess the sensitivity of the results to the length of the simulated time interval the time-averaged and standard deviation of the key parameters was evaluated for simulated periods ranging from 15 to about 65 convective times. It was observed that between 50 and 65 convective times the averaged PR across these range of simulation periods varied by less than $0.1 \%$ and the standard deviation of PR changed by $2.5 \%$. Similarly for the distortion metrics, the time-averaged DC60, CDI, RDI and SI all changed by less than $1 \%$ and the standard deviation varied by less than $2.5 \%$ between 50 and 65 convective times. A simulation length of 55 convective times was used for the statistical and spectral analysis as well as for the POD assessment.

\section{E. Data processing}

It is typical in intake distortion experimental work to evaluate the flow field at the AIP using a total pressure rake which comprises 8 circumferential rakes each with 5 radial probes. The probes are positioned across the AIP so that they are appropriately spaced for area-averaging purposes. Although the computational studies provide a substantially greater spatial resolution, to enable assessment within the established context some of the integral results presented herein the data is sampled at 40 individual locations to enable comparisons with the experimental data which was acquired on a conventional $8 \times 5$ rake. For the flow field spectral and POD analysis, the full computed flow field is used. A spectral analysis of the flow field at the AIP is performed and the frequency content is nondimensionalized as Strouhal number (St) based on the AIP diameter and area-average axial velocity $\left(S t=f D_{A I P} / \overline{W_{A I P}}\right)$. Six St intervals of about 0.14 are defined from frequency intervals of $100 \mathrm{~Hz}$ based on the AIP diameter and the axial velocity from S-Duct 2A case. For a better understanding of the nature of the flow-field oscillations, the spectral analysis is encompassed with coherence and phase analysis, obtained from the Fourier 
transform of the cross-correlation of the total pressure at two distinct points at the AIP assessed across the time history of the simulation.

\section{Results}

\section{A. Overall flowfield and comparison with experimental results}

The unsteady flow in the duct is predominately affected by the flow separation along the duct centre line and the development of the classical secondary flows. A qualitative exposition of the DDES calculated flow field is highlighted in Fig. 2 which shows a snapshot of $\mathrm{x}$-vorticity $\left(\Omega_{x}^{*}=\Omega_{x} D_{A I P} / \overline{W_{A I P}}\right)$ and z-vorticity $\left(\Omega_{z}^{*}=\Omega_{z} D_{A I P} / \overline{W_{A I P}}\right)$ on the y-z symmetry plane of the duct. The distributions of $\Omega_{x}^{*}$ (Fig. 2a) show the separation of the boundary layer after the first part of the S-Duct and the subsequent evolution of the shear layer. The streamwise vortices which are associated with the secondary flows are also unsteady and have a considerable impact on the separated flow field. This is evident in the snapshots of $\Omega_{z}^{*}$ (Fig. 2b) which, along with the $\Omega_{x}^{*}$ distributions, also illustrate the large extent of the downstream duct which is affected by the unsteady flow distortions. Fig. 3 shows a snapshot of a $\mathrm{Q}$-criterion iso-surface $\left(\mathrm{Q}=1 \times 10^{7}\right)$ coloured by contours of the streamwise $\mathrm{z}$-velocity. This provides a further qualitative illustration of the separated flow field which arises after the first part of the duct and the coherent structures that are resolved by the DDES simulations.

The numerical methods are validated using the configurations S-Duct 2AS and 2BS (Table 2) based on the quantitative experimental data provided in Delot et $\mathrm{al}^{9,12}$. The computational data has been linearly interpolated to the location of the 40 experimental data points for a consistent comparison. A comparison of the static distortion obtained from the time-averaged flow field is presented in Table 4. For both the RANS and DDES calculations, the pressure recovery is within $0.1 \%$ of the measurements for the high Mach number case S-Duct 2AS and low Mach number case $2 \mathrm{BS}$. Overall the other static distortion metrics calculated with DDES are typically within $15 \%$ of the reported measured values for the low Mach number configuration (2BS), and about 20\% for the high Mach number (2AS). In all cases the DDES results are greater than the measurements. For the RANS calculations, the static distortion metrics are notably worse than the DDES results, with a large range of differences from the measurements. In particular, the RANS calculated circumferential distortion metrics have differences of about $20 \%$ to $65 \%$ compared with the measurements. 
The distribution of the pressure recovery at the $\mathrm{AIP}_{\exp }$ similarly shows good agreement in the levels and extent of the total pressure loss regions for high Mach number 2AS (Fig. 4). Similar results were found for low Mach number 2BS. The DDES calculations show the characteristic loss region at the lower duct sector although the simulations slightly over estimate the loss relative to the measurements. Although the distribution of total pressure ratio is very similar between the CFD and experiment and the overall loss is identical, the differences in the distortion metrics are a little higher. The CFD results are symmetric although the experimental data shows a slight asymmetry in the upper regions most probably associated with the experimental uncertainty. A key element of the current work is in the unsteady aerodynamics and for case 2AS both the computations and measurements show peak unsteadiness in two symmetric lobes in the lower sector (Fig. 5). In these peak lobes the measured total pressure ratio standard deviation is about 3.0\% whereas the DDES simulations indicate a level of about 3.2\%. A spectral analysis of total pressure from the computational and experimental data highlights the flow characteristics at different frequencies (Fig. 6). Overall there are distinct regions in which the total pressure fluctuations have clear dominant distributions at particular frequency bands and there are some notable aspects evident in both the computational and experimental data. At the frequency of $100-200 \mathrm{~Hz}$ both the CFD and experiments indicate a local maximum unsteadiness in the regions associated with the time-averaged positions of the primary contra-rotating secondary vortices. The CFD has slightly higher levels of unsteadiness although the topology is in good agreement with the measurements. At the higher frequency bands the unsteadiness becomes centrally positioned and the levels gradually decay. 

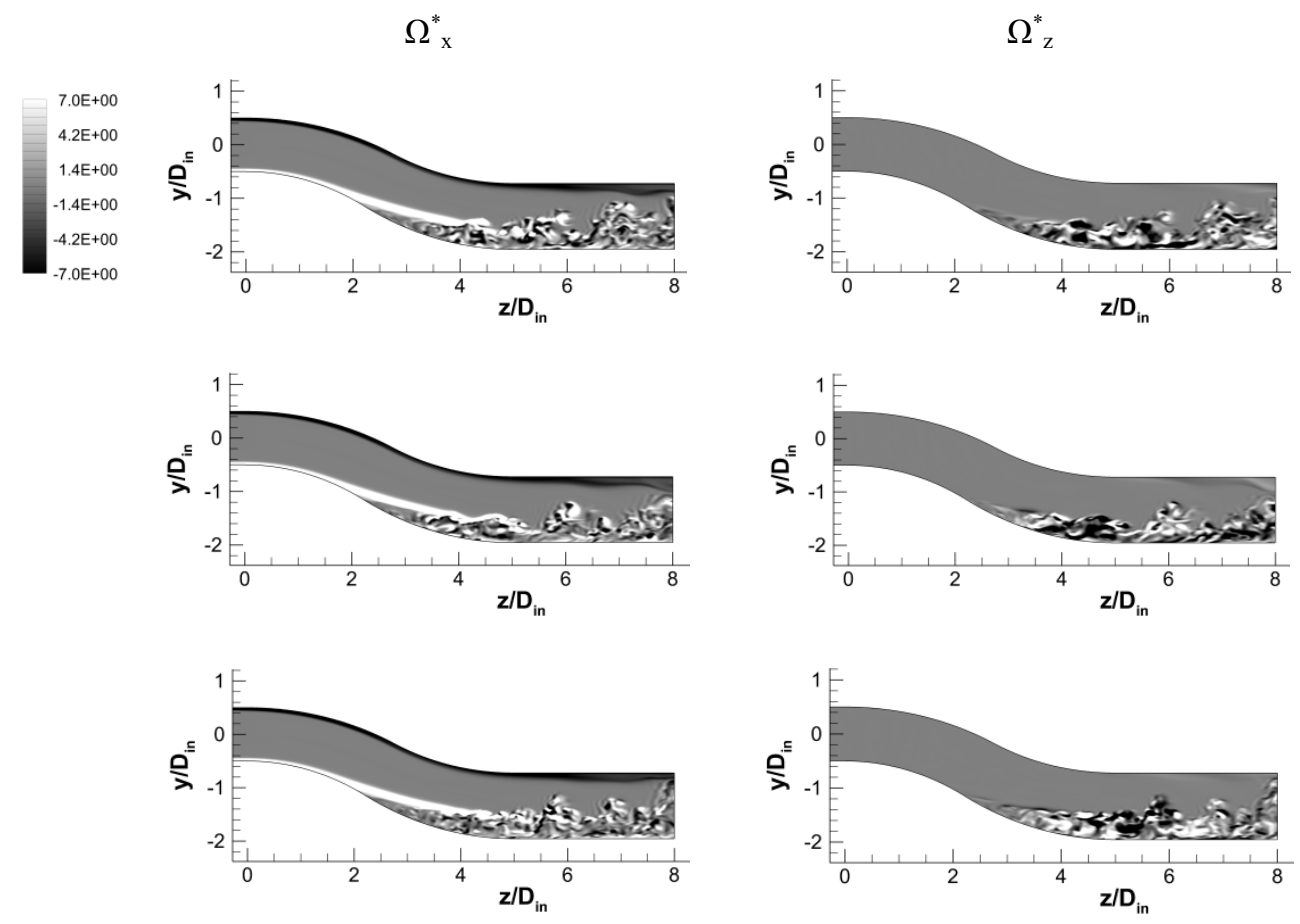

Fig. 2 Snapshots of $x$ - and z-vorticity on the z-y symmetric plane. Duct-2A, fine mesh.

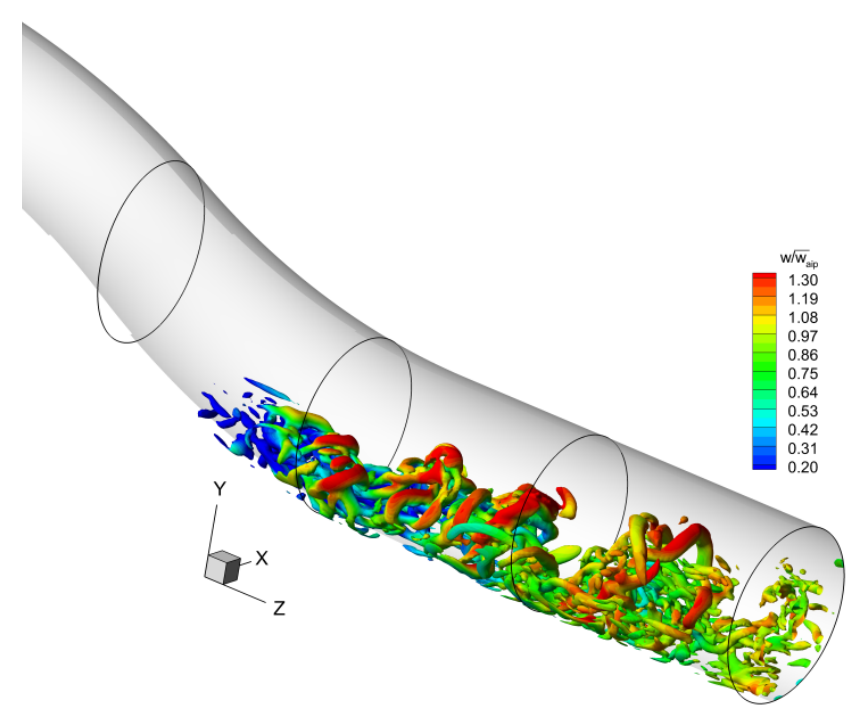

Fig. 3 Snapshot of a $Q$-criterion iso-surface $\left(Q=1 \times 10^{7}\right)$ in the duct exit coloured by streamwise velocity $\left(\mathrm{w} / \overline{w_{A I P}}\right)$. Duct-2A, fine mesh. 
Table 4 Comparison of computated and measured ${ }^{9}$ static distortion for S-Duct 2 AS and 2 BS

\begin{tabular}{|c|c|c|c|c|c|c|c|}
\hline S-duct & $\overline{P R}_{<p 0>}$ & $D C 60_{<p 0}=$ & $C D I_{<p 0>}$ & ${\overline{\Delta P_{C} / P}}_{<p 0>}$ & $\max \left(\Delta P_{C} / P\right)_{<p 0>}$ & $R D I_{<p 0>}$ & $\overline{S I}_{<p 0>} \operatorname{deg}$ \\
\hline 2AS RANS & 0.970 & 0.535 & 0.0585 & 0.0326 & 0.0409 & 0.0189 & 2.23 \\
\hline 2AS DDES & 0.971 & 0.415 & 0.0579 & 0.0238 & 0.0311 & 0.0307 & 2.08 \\
\hline 2AS Exp & 0.971 & - & 0.0483 & 0.0195 & 0.0252 & 0.0251 & - \\
\hline 2BS RANS & 0.994 & 0.409 & 0.0160 & 0.0072 & 0.0088 & 0.0067 & 2.17 \\
\hline 2BS DDES & 0.993 & 0.327 & 0.0143 & 0.0060 & 0.0074 & 0.0083 & 2.01 \\
\hline 2BS Exp & 0.993 & - & 0.0126 & 0.0051 & 0.0065 & 0.0070 & - \\
\hline
\end{tabular}
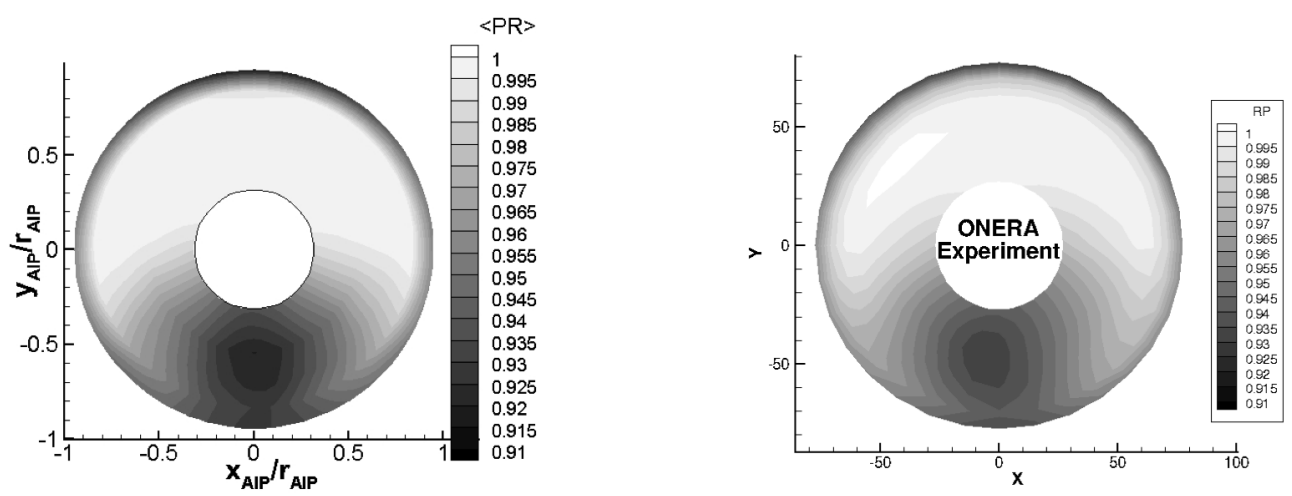

Fig. 4 Time-averaged pressure recovery S-Duct 2AS. DDES (left) and measurements (right) ${ }^{12}$.
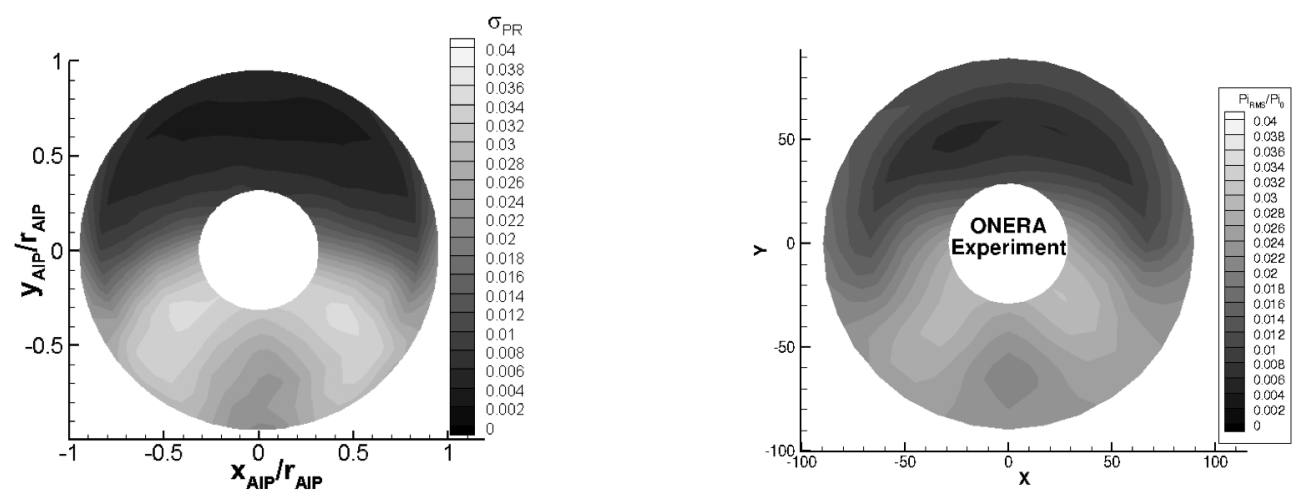

Fig. 5 Pressure recovery standard deviation S-Duct 2AS. DDES (left) and experiment (right) ${ }^{12}$. 


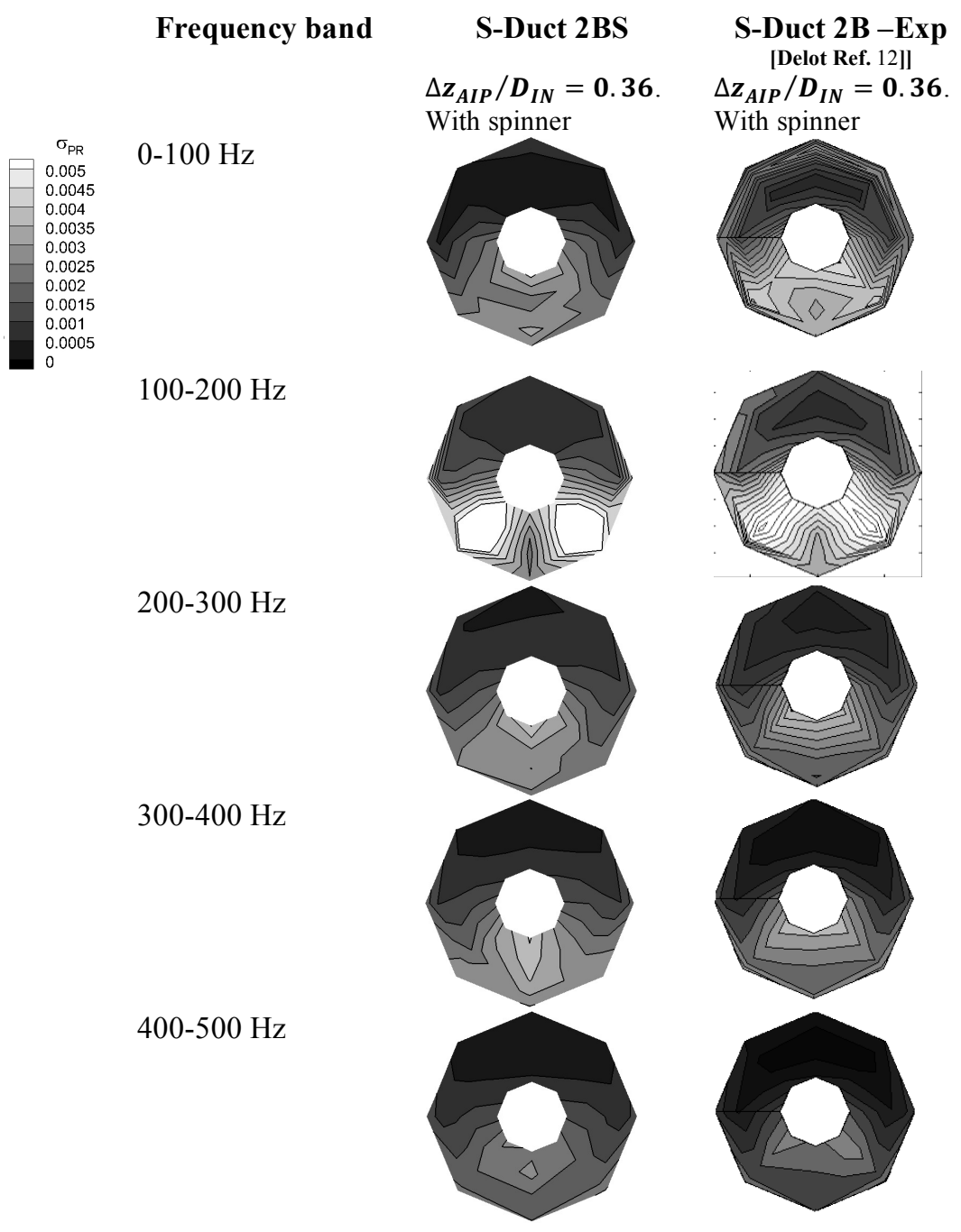

Fig. 6 Comparison of total pressure spectral bands for S-Duct 2BS DDES calculations (left) and measurements (right) ${ }^{12}$ at the $\mathrm{AIP}_{\text {exp. }}$.

\section{B. Time-averaged and statistical parameters}

For the five configurations without the spinner considered in this study (Table 2), values of the key static distortion descriptors evaluated from the time-averaged flow field are calculated at the AIP (Fig. 1a), which is located $0.5 \mathrm{D}_{\text {in }}$ downstream the S-Duct outlet. For completeness, a comparison with the results from the RANS simulations is also provided (Table 5). Not surprisingly the average pressure recovery is lower for the S-Duct 3 configurations due to the higher offset, and there is a slight benefit of the high Reynolds number when comparing SDuct 1 and S-Duct 2A. The DDES DC60 levels are higher for the S-Duct 2 configurations when compared with S- 
Duct 3 which has a higher offset. This is because for the S-Duct 3 cases although the loss in total pressure is greater and over a larger region (Fig. 7), it is more centrally located and the DC60 parameter is constructed so that this results in a lower measure of distortion. It is also notable that there is a substantial difference in the DC60 levels between the RANS and DDES calculations for S-Duct 2 with the RANS levels being notably higher. S-Duct 3 shows fewer differences and the RANS calculations are broadly in good agreement with the DDES. CDI and RDI are less sensitive to the differences between the RANS and DDES although the differences generally increase with Mach number. It is also of note that the RANS calculated swirl intensity for S-Duct 3 is almost twice the level of the DDES solutions. Overall it indicates that even for the time-averaged properties, although the average PR may be calculated relatively well by the RANS simulations, there can be notable differences in the more complex distortion descriptors.

Fig. 7 illustrates the time-averaged and standard deviation distribution of the swirl angle $\alpha$ at the AIP section (Fig. 1) based on the full CFD spatial resolution. Notable aspects are observed due to the effect of the nondimensional S-duct offset when $\mathrm{H}_{\mathrm{o}} / \mathrm{L}$ is increased from 0.268 (S-Duct 2) to 0.493 (S-Duct 3). The time-averaged location of the twin vortices moves toward the central part of the AIP and their effect is extended to an upper region of the plane. The main total pressure loss region also moves to a more central position and the region of maximum total pressure unsteadiness is mainly associated with the upper bounds of this loss region for both S-Duct 2 and 3. For the higher offset configuration (S-Duct 3), the peak value of $\langle\alpha\rangle$ increases from $12^{\circ}$ to $20^{\circ}$. However, there is a slight reduction in the swirl fluctuations $\sigma_{\alpha}$ for S-Duct 3 and the distribution is more centrally positioned. The reduction in unsteady swirl for the high-offset duct is of note as it is different from the effect on total pressure and, due to the radial movement of the main streamwise vortices, the centreline separation and the secondary flow vortices become more intertwined with a damping of the swirl unsteadiness. 


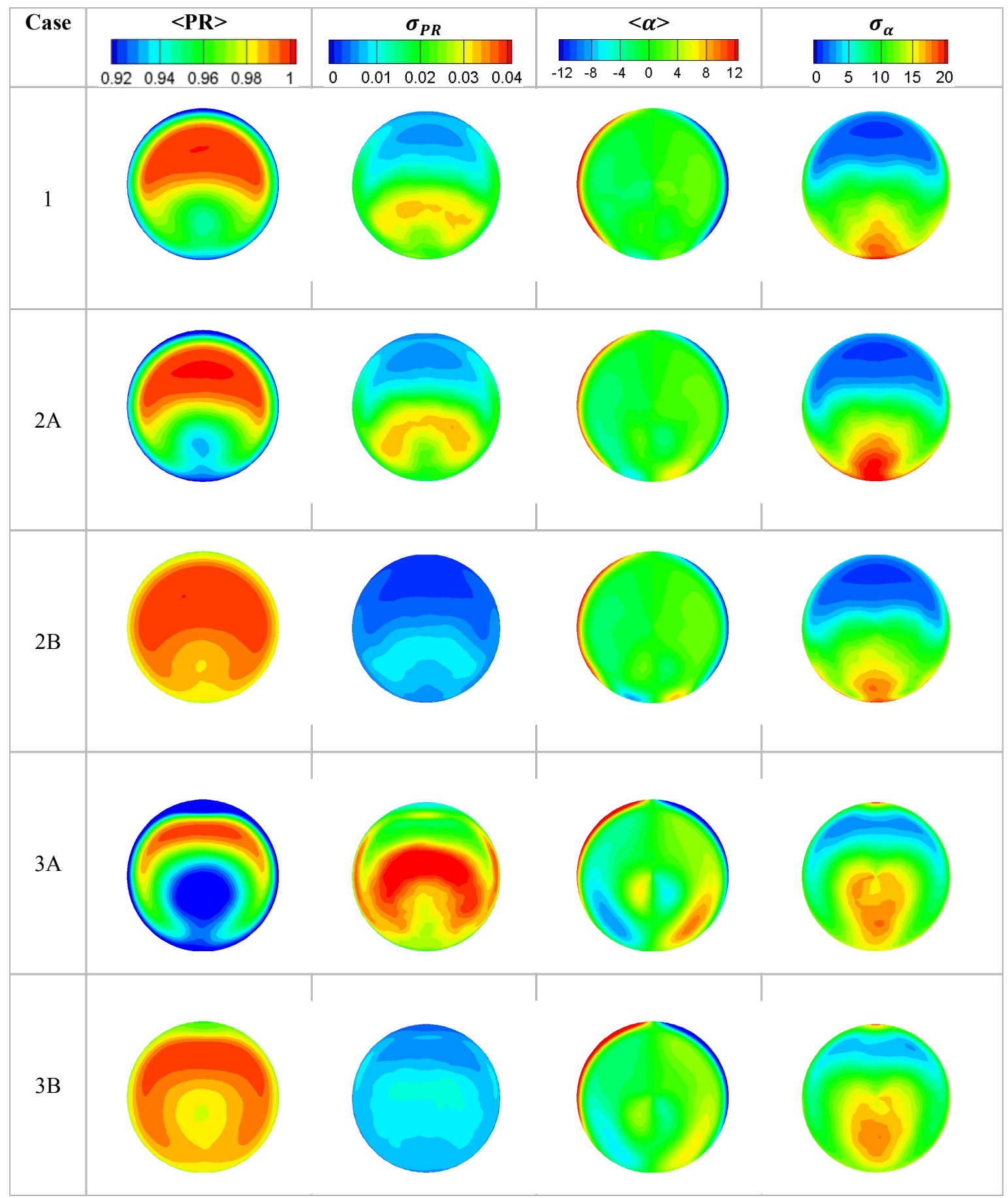

Fig. 7 Calculated pressure recovery and swirl at the AIP section. Time-averaged and standard deviation. 
Table 5 Summary of time-averaged static performance and static distortion metrics at the AIP section.

\begin{tabular}{|c|c|c|c|c|c|c|c|}
\hline S-Duct & $\overline{P R}_{<p 0>}$ & $D C 60_{<p 0>}$ & $C D I_{<p 0>}$ & $R D I_{<p 0>}$ & ${\overline{\Delta P_{C}} / P_{<p 0>}}$ & $\max \left(\Delta P_{C} / P\right)_{<p 0>}$ & $\overline{S I}_{<\alpha>}$ \\
\hline $1 \mathrm{DDES}$ & 0.973 & 0.265 & 0.036 & 0.033 & 0.015 & 0.020 & 1.86 \\
\hline 1 RANS & 0.970 & 0.493 & 0.059 & 0.021 & 0.028 & 0.037 & 2.09 \\
\hline 2A DDES & 0.969 & 0.354 & 0.047 & 0.032 & 0.019 & 0.025 & 1.77 \\
\hline 2A RANS & 0.968 & 0.519 & 0.060 & 0.022 & 0.030 & 0.040 & 2.31 \\
\hline 2B DDES & 0.993 & 0.280 & 0.011 & 0.009 & 0.004 & 0.005 & 1.86 \\
\hline 2B RANS & 0.992 & 0.466 & 0.016 & 0.006 & 0.007 & 0.010 & 2.14 \\
\hline 3A DDES & 0.947 & 0.242 & 0.058 & 0.030 & 0.024 & 0.034 & 3.12 \\
\hline 3A RANS & 0.947 & 0.175 & 0.059 & 0.040 & 0.027 & 0.037 & 6.02 \\
\hline 3B DDES & 0.988 & 0.156 & 0.013 & 0.008 & 0.005 & 0.007 & 2.59 \\
\hline 3B RANS & 0.988 & 0.159 & 0.014 & 0.008 & 0.006 & 0.008 & 5.73 \\
\hline
\end{tabular}

\section{Flow distortion characteristics}

In this section the distortion descriptors have been evaluated from the flow field at each time step , and the respective time-signal statistics are summarized in Table 6 . The high level of unsteady swirl are notable with timeaveraged SI in the region of $6-9^{\circ}$ but with an area averaged maximum SI of up to $16.4^{\circ}$ for S-Duct $3 \mathrm{~A}$. The effect of Mach number for both S-Duct 2 and 3, shows that there is a large increase in the steady and unsteady CDI, RDI and $\overline{\Delta \boldsymbol{P}_{C} / \boldsymbol{P}}$ with $\mathrm{M}$, but that in general the other parameters are unaffected. The most notable changes arise due to the change in geometry from S-Duct 2 to the higher offset S-Duct 3. Interestingly the $<$ DC60 $>$ reduces for the higher offset but the swirl based parameters $<$ SC60 $>$ and SI increase. Broadly CDI and RDI are unaffected which highlights the limitations of only considering time-averaged total pressure based distortion metrics. The most notable impact on the unsteady distortion is at the higher Mach number (2A and $3 \mathrm{~A})$ where the deviation of RDI doubles with the increase in offset. Overall these results highlight the importance of considering both the unsteady distortion aspects as well as the swirl based descriptors. The effect of Reynolds number shows that there is generally a very small reduction in the unsteady distortion metrics when the Reynolds number is reduced from $2.6 \times 10^{6}$ to $1.7 \times 10^{6}$ for S-Duct 1 and S-Duct $2 \mathrm{~A}$.

Although the statistics of the key distortion descriptors RDI and CDI (Table 6) highlight significant differences between the different duct configurations, it is also useful to consider the unsteady aspects of these metrics in more detail. Within this context, the simultaneous distributions of RDI and CDI are of interest (Fig. 8). For S-Duct 1 there is a relatively clustered distribution of points in a range of variation of CDI of about 0.09 and a variation of RDI of 
about 0.03 . In comparison with the datum S-Duct 1 configuration, $\mathrm{S}$-Duct $2 \mathrm{~A}$ is the same geometric shape but at a lower inlet diameter and therefore the Reynolds number $\left(\operatorname{Re}_{\operatorname{Din}}=1.7 \times 10^{6}\right)$ is also reduced. Overall the RDI-CDI map is similar, but the range of CDI is slightly truncated and it is also of interest to note the excursion of occasional instances of relatively high values which are over twice the time-averaged value. For Duct 2B where the Mach number is reduced from 0.36 to 0.18 , there is a dramatic reduction in the distortion coefficients where they are generally 3 to 4 times smaller than the results for S-Duct $2 \mathrm{~A}$ at $\mathrm{M}=0.36$ due to the nature of the definition of CDI and RDI and the notable change in $<\mathrm{PR}>$. For the S-Duct 3 configuration where the S-duct offset $\mathrm{H}_{0} / \mathrm{L}$ is increased from 0.268 to 0.493 , there is an increase in both the time-averaged RDI and CDI as well as in the extreme values. With this high-offset duct, there is more notable scatter in the RDI distribution in particular where the standard deviation of RDI has almost doubled from approximately 0.006 to 0.013 . These extreme distortions are of interest as these events may be a key design consideration in the response of the compression system and the ultimate effect on surge margin.

The nature of the unsteady aspects on the swirl and total pressure distortions is illustrated in the SC60-DC60 maps (Fig. 8). For S-Duct 1 and S-Ducts 2A and 2B, both the SC60 and DC60 show notable levels of variation relative to the mean values and clearly highlight the low frequency, but high distortion, events that occasionally occur. For example, S-Duct 2A shows that the peak SC60 and DC60 are about twice the mean levels. The distributions of SC60 and DC60 highlight the importance of considering both the swirl and pressure distortions simultaneously. For example, at a mean $<$ SC60 $>$ of around 0.2 the DC60 excursions range from about 0.15 to 0.75 . Similarly, the SC60 ranges from about 0.1 to 0.4 for a mean $<$ DC60 $>$ of 0.43 . The impact of the higher offset for SDuct 3A resulted in an increase in the unsteady RDI. In contrast, for this high-offset duct the unsteady aspects of the SC60 and DC60 metrics are notably reduced. This is partially reflected in Fig. 7, where the unsteady swirl takes a more central position and the levels are reduced relative to the low-offset S-Duct 2. The same characteristics and differences are observed at both Mach numbers. Overall it highlights the complexity of the problem, the importance of both the steady and unsteady aspects, and the importance of both swirl and pressure based metrics. 
Table 6 Summary of DDES calculated distortion statistics

\begin{tabular}{rccccccc}
\hline \hline & PR & CDI & $\overline{\Delta \boldsymbol{P}_{\boldsymbol{C}} / \boldsymbol{P}}$ & RDI & $\mathbf{D C}(\mathbf{6 0 )}$ & $\mathbf{S C}(\mathbf{6 0 )}$ & $\overline{\mathbf{S I}} \mathbf{\text { deg}}$ \\
\hline S-Duct 1 & & & & & & & \\
$<.>$ & 0.973 & 0.059 & 0.023 & 0.033 & 0.348 & 0.198 & 6.0 \\
$\sigma$. & 0.002 & 0.019 & 0.009 & 0.006 & 0.149 & 0.059 & 1.9 \\
peak & 0.984 & 0.118 & 0.050 & 0.054 & 0.781 & 0.470 & 14.0 \\
\hline S-Duct 2A & & & & & & & \\
$<.>$ & 0.969 & 0.066 & 0.027 & 0.032 & 0.426 & 0.207 & 6.2 \\
$\sigma$. & 0.003 & 0.015 & 0.008 & 0.006 & 0.131 & 0.052 & 1.7 \\
peak & 0.975 & 0.116 & 0.052 & 0.051 & 0.849 & 0.410 & 13.1 \\
\hline S-Duct 2B & & & & & & & \\
$<.>$ & 0.993 & 0.017 & 0.007 & 0.009 & 0.362 & 0.193 & 5.8 \\
$\sigma$ & 0.001 & 0.005 & 0.002 & 0.002 & 0.144 & 0.057 & 1.8 \\
peak & 0.995 & 0.033 & 0.013 & 0.013 & 0.732 & 0.436 & 12.7 \\
\hline S-Duct 3A & & & & & & & \\
$<.>$ & 0.947 & 0.077 & 0.033 & 0.036 & 0.289 & 0.243 & 8.3 \\
$\sigma$. & 0.003 & 0.014 & 0.006 & 0.013 & 0.099 & 0.047 & 1.8 \\
peak & 0.963 & 0.137 & 0.052 & 0.101 & 0.668 & 0.521 & 16.4 \\
\hline S-Duct 3B & & & & & & & \\
$<.>$ & 0.988 & 0.018 & 0.007 & 0.009 & 0.233 & 0.243 & 8.5 \\
$\sigma$. & 0.992 & 0.004 & 0.001 & 0.002 & 0.091 & 0.045 & 1.8 \\
peak & 0.015 & 0.033 & 0.012 & 0.027 & 0.602 & 0.388 & 14.8 \\
\hline
\end{tabular}



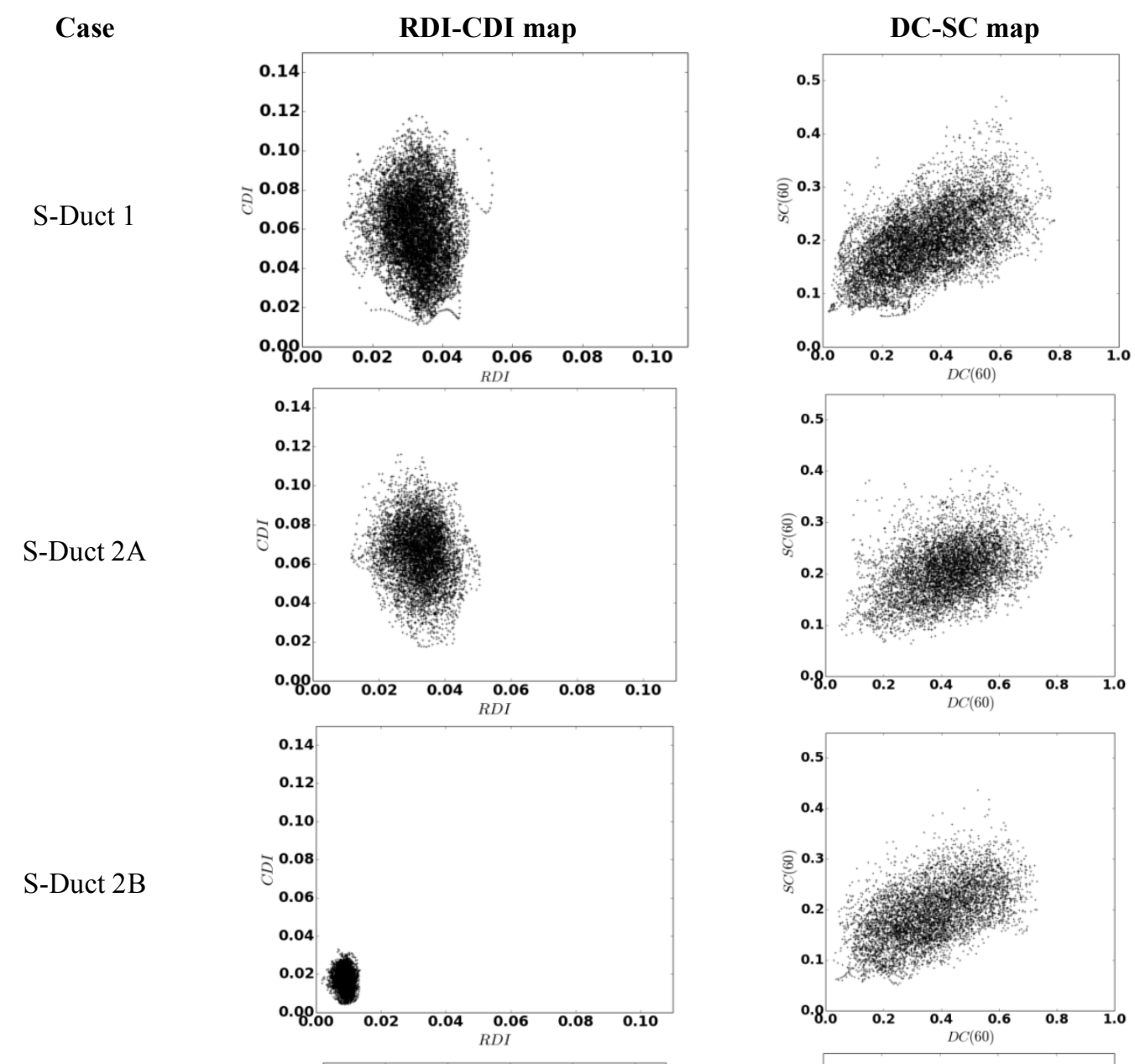

S-Duct 3A
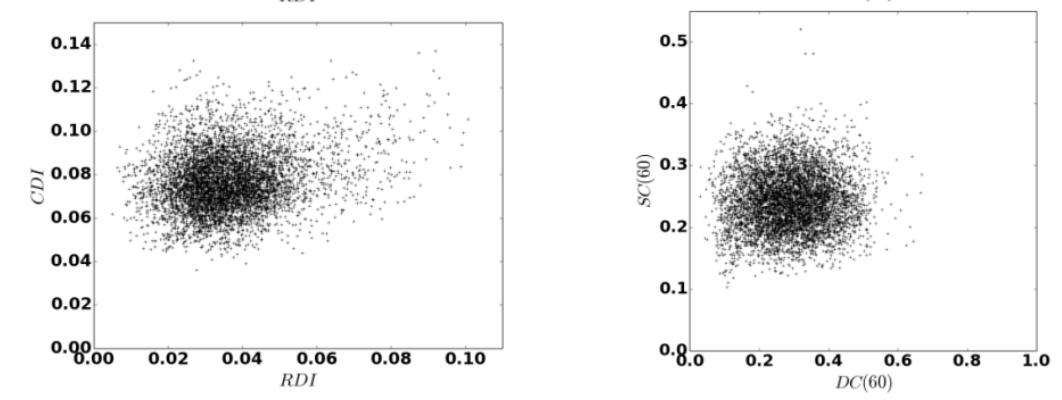

Fig. 8 Distortion metric maps from DDES temporal snapshots at the AIP section

\section{Flowfield Spectral Analysis at the AIP}

For the DDES calculations, the unsteady flow field at the AIP has been analysed and the distribution of the total pressure fluctuations $\sigma_{\mathrm{PR}}$ divided in Strouhal number bands of about 0.143 presented for S-Duct 1, S-Duct 2 and SDuct 3 (Fig. 9). For S-Ducts 1, 2A and 2B, the most dominant feature is in the Strouhal range of 0.29 to 0.43 , where there are the two distinct symmetric peaks in the region around the time-averaged location of the classical 
streamwise secondary flow vortices. These regions of high unsteadiness were previously measured by Delot et al. ${ }^{12}$ for the S-Duct 2B case across a similar Strouhal number range. The second most notable aspect is the relatively high levels of unsteadiness in the centre of the AIP which arises at $\mathrm{St}=0.57-0.72$ and $\mathrm{St}=0.72-0.86$, for S-Ducts $2 \mathrm{~A}$ and $2 \mathrm{~B}$, respectively. This higher frequency unsteadiness is more associated with the boundary layer separation which arises from the first bend of the S-Ducts. Similar characteristics were observed in measurements reported by Delot et al. ${ }^{12}$ for the S-Duct $2 \mathrm{~B}$ for a frequency band between $\mathrm{St}=0.55-0.82$.

For S-Ducts $3 \mathrm{~A}$ and $3 \mathrm{~B}$, the offset has increased from $\mathrm{H}_{\mathrm{o}} / \mathrm{L}=0.268$ to 0.493 and the unsteady flowfield distributions are different. In general the unsteadiness is more centrally located in agreement with the changes observed for the time-averaged total pressure ratio (Fig. 7) and the main unsteadiness arises at a higher St range across $0.43-0.72$. These more central regions of high fluctuations observed for S-Duct 3B within the frequency band of $\mathrm{St}=0.43-0.72$ were similarly reported from measurements by Garnier ${ }^{11}$ across a frequency band of $\mathrm{St}=0.40-0.70$, for the same geometry at similar flow conditions. The characteristics associated with the symmetric secondary flows are still evident, but are much less distinct. The changes in Reynolds number and Mach number change the level of unsteadiness, but do not have a notable impact on the Strouhal number distributions or characteristics. Overall the results indicate that the main total pressure unsteadiness is associated with changes in the secondary flow vortices as well as regions associated with the central streamwise separation. The indications are that at modest offset these events arise at different frequency bands, but that as the duct offset increases the demarcation between these aspects becomes less clear.

To help to understand the underlying nature of the flow unsteadiness, an assessment of the cross correlation within different parts of the flow field has been considered. For S-Duct 2A, a cross-correlation of the unsteady total pressure at the peak location of unsteadiness associated with the time-averaged vortex positions has been performed (Fig. 10). The coherence shows that there are two main frequencies of interest at approximately $260 \mathrm{~Hz}$ and $540 \mathrm{~Hz}$, which correspond to a St of about 0.4 and 0.8 respectively. The phase characteristic shows that the unsteadiness at $260 \mathrm{~Hz}$ is approximately $-180^{\circ}$ out of phase while the unsteadiness at $540 \mathrm{~Hz}$ is relatively in phase. Taken in conjunction with the flow field topology this indicates that there is a circumferential variation associated with the secondary flows as well as a different mechanism associated with the high frequency in-phase unsteadiness in the centre of the AIP. Analysis of the flow field indicates that this is associated with the streamwise flow separation from the first S-Duct bend. Similar results were found for S-Duct 2B with the pertinent Strouhal numbers slightly 
reduced to 0.35 and 0.7 . For S-Duct 3, the spectra are more distributed and the phase characteristics are not as distinct (Fig. 11). There is a maximum in the range $400-500 \mathrm{~Hz}$, with a St around 0.6 , but there is a notable level of more broadband unsteadiness. In this context it is surmised that secondary flows of the high-offset S-Duct 3 are still present but are positioned in a more central location and are more merged with the unsteadiness associated with the centreline separations. 


\section{S-Duct 1}

$\mathrm{M}=0.36$

$\mathrm{Re}_{\mathrm{D}}=2.6 \times 10^{6}$

$\mathrm{H}_{\mathrm{o}} / \mathrm{L}=0.268$

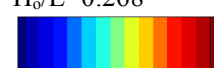

S-Duct 2A

$\mathrm{M}=0.36$

$\mathrm{Re}_{\mathrm{D}}=1.7 \times 10^{6}$

$\mathrm{H}_{\mathrm{o}} / \mathrm{L}=0.268$

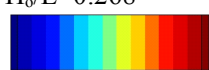

S-Duct 2B

$\mathrm{M}=0.18$

$\mathrm{Re}_{\mathrm{D}}=1.1 \times 10^{6}$

$\mathrm{H}_{\mathrm{o}} / \mathrm{L}=0.268$
S-Duct 3A

$\mathrm{M}=0.39$

$\mathrm{Re}_{\mathrm{D}}=1.8 \times 10^{6}$

$\mathrm{H}_{\mathrm{o}} / \mathrm{L}=0.493$

S-Duct 3B

$\mathrm{M}=0.18$

$\mathrm{Re}_{\mathrm{D}}=1.1 \times 10^{6}$

$\mathrm{H}_{\mathrm{o}} / \mathrm{L}=0.493$

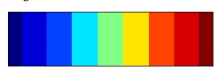

$0 \quad 0.026$

0
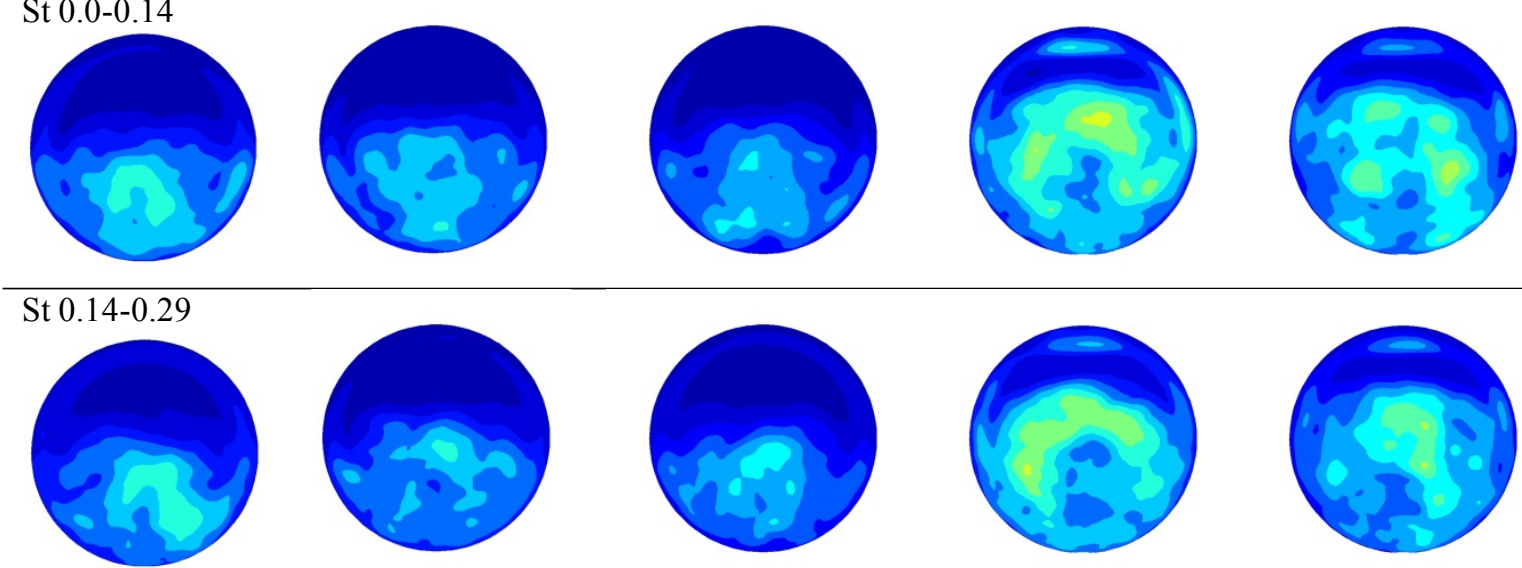

St 0.29-0.43
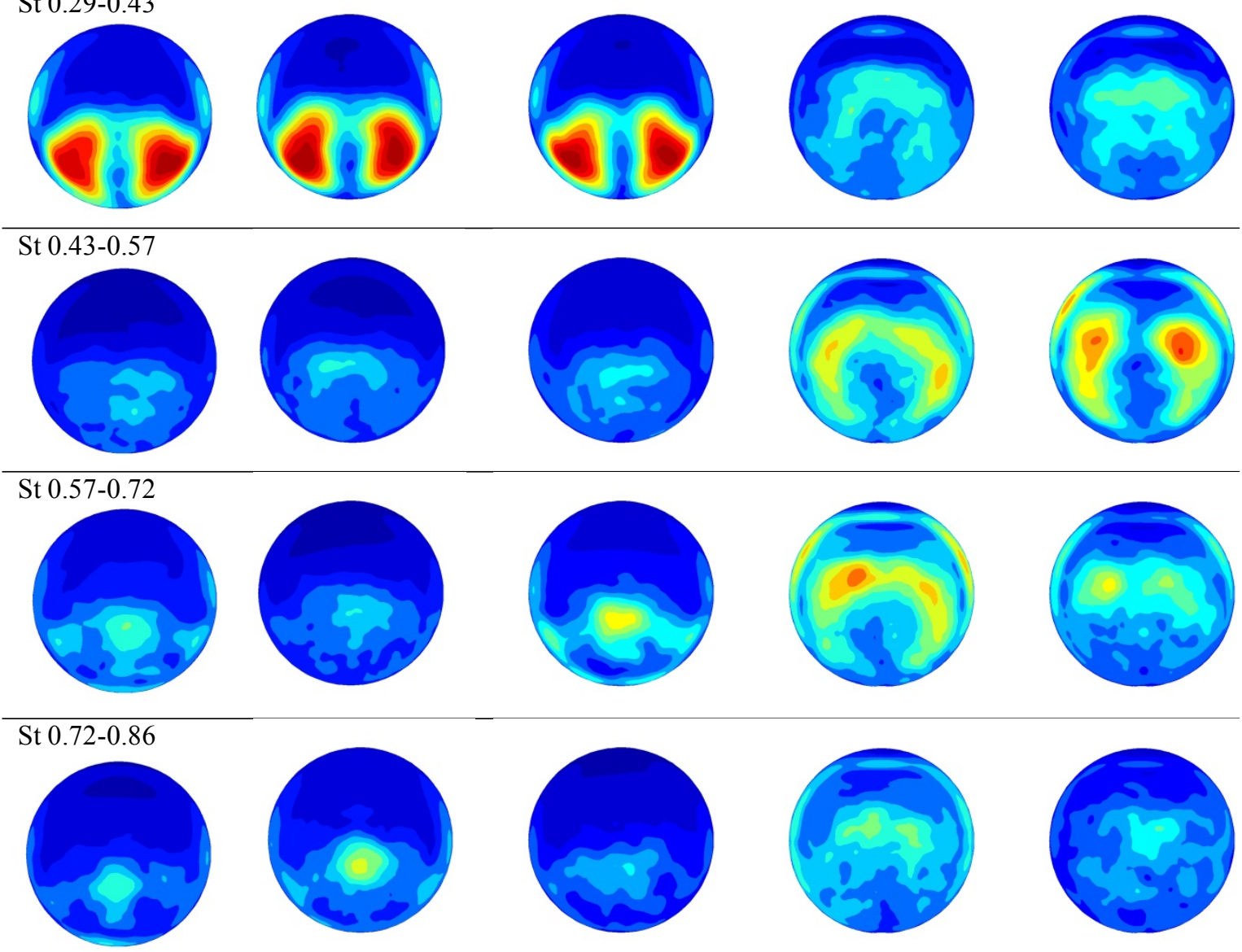

Fig. 9 AIP spectral distribution of total pressure fluctuations. Note the different contour scales for S-Duct 2B and 3B. 

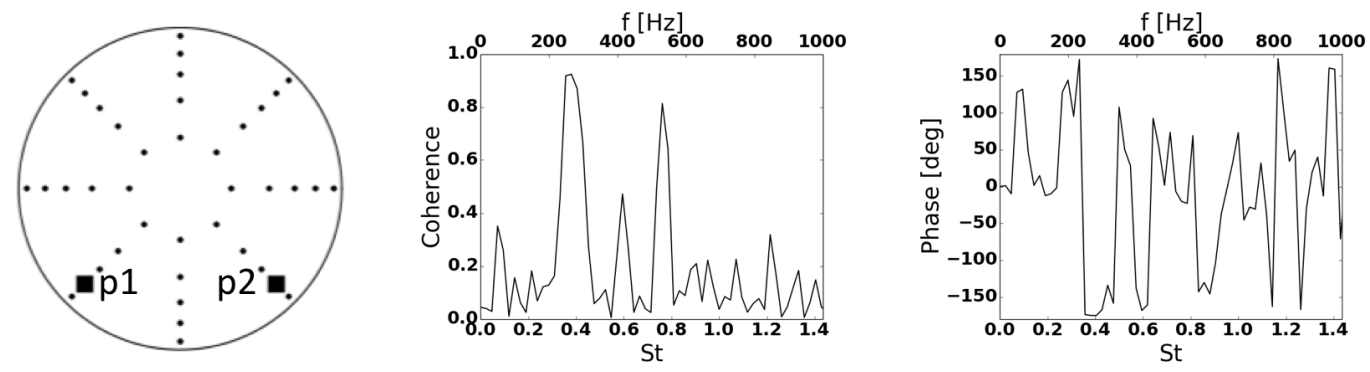

Fig. 10 Coherence and phase distribution between two opposite points on the AIP. S-Duct $2 \mathrm{~A}$.
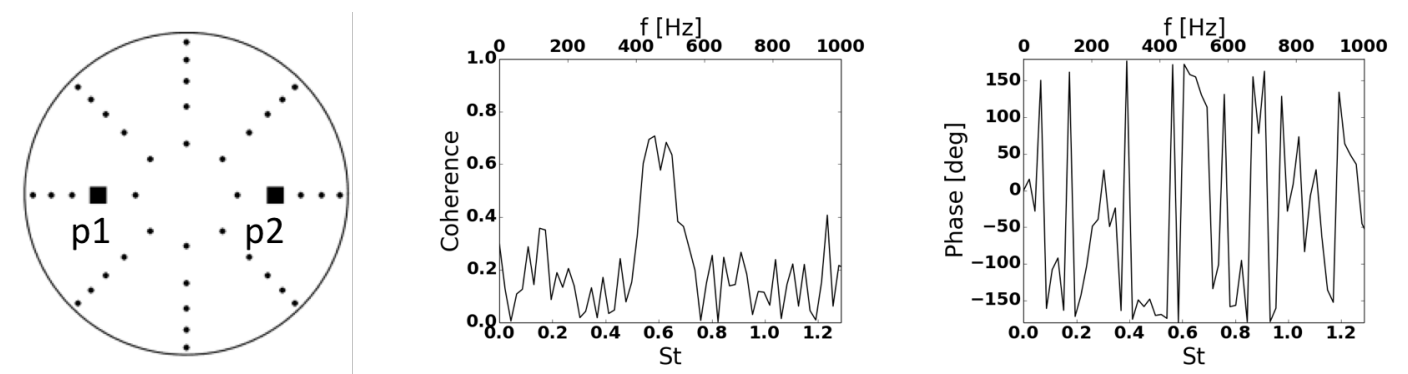

Fig. 11 Coherence and phase distribution between two opposite points on the AIP. S-Duct 3A.

\section{E. Proper Orthogonal Decomposition}

To develop a better understanding of the unsteady behaviour of the flow and the underlying flow structures, the use of the Proper Orthogonal Decomposition (POD) method was applied to the total pressure distribution at the AIP. The modes are sorted by the energetic content of their signal in descending order.

\section{POD method}

Proper Orthogonal Decomposition (POD) has been widely utilised to extract dominant flow features and ultimately coherent structures ${ }^{29}$. The POD has been recently applied for the analysis of turbulent flows in different applications, such as the flow in a backward-facing step ${ }^{29,30}$, reciprocating internal combustion engines ${ }^{31}$, turbulent boundary layers ${ }^{32}$ and curved pipes ${ }^{33}$.A detailed mathematical description is beyond the scope of this paper, and only a brief review of the key aspects of the POD is presented. For a more expansive derivation, the reader is referred to Holmes et $\mathrm{al}^{34}$. Considering for simplicity a scalar field $q(x, y, t)$, the POD consists of finding an orthogonal basis $\left\{\varphi_{j}(x, y)\right\}$ that maximises the projection of $u$ onto $\varphi$. The functions $\varphi_{j}$, often called POD characteristic modes, 
represent those flow features responsible for most of the signal energy in a statistical sense and may be, sometimes, associated with coherent structures. The POD representation of the original flow field is then obtained by a series of the POD characteristic modes, each of them being multiplied by a temporal coefficient ${ }^{35}$ (Eq. 21)

$$
q_{k}(x, y, t)=\sum_{j=1}^{k} a_{j}(t) \varphi_{j}(x, y)
$$

The temporal coefficients are statistically uncorrelated and the modes are orthonormal. These properties permit to express the area-averaged mean-squared value of the scalar field as the series of the mean-squared value of the temporal coefficients (Eq. 22).

$$
\left\langle\overline{q_{k}^{2}}\right\rangle=\sum_{j=1}^{k}\left\langle a_{j}^{2}\right\rangle
$$

To increase convergence of the reconstruction, the modal contributions in the series are ordered in decreasing order of the mean-square value of the associated temporal coefficient, which is often referred to as the modal energy. If the variable of choice is the velocity vector field, then the POD maximises the kinetic energy content in the reconstruction ${ }^{36}$. In the present investigation the POD has been implemented using the method of snapshots ${ }^{37}$, which consists of obtaining the POD modes as $\varphi_{j}=\left\langle q \beta_{j}\right\rangle$, where $\beta_{j}$ are the eigenfunctions of the correlation matrix, $\mathrm{C}\left(\mathrm{t}, \mathrm{t}^{\prime}\right)$ (Eq. 23). The temporal coefficients are then evaluated by projection of each mode, properly normalised, onto each snapshot of the original flow field (Eq. 24).

$$
\begin{gathered}
C\left(t, t^{\prime}\right)=\frac{1}{A} \int_{A} q\left(x, y, t^{\prime}\right) q(x, y, t) d A \\
a_{j}(t)=\frac{1}{A} \int_{A} \varphi_{j}(x, y) q(x, y, t) d A
\end{gathered}
$$

\section{POD analysis for total pressure}

For S-Duct 2 and S-Duct 3 at both Mach numbers the distribution of the variance for the POD mode coefficients "a", as a percentage of the total variance, are dominated by the first 3 modes with a subsequent gradual monotonic decrease at higher modes. The relative importance of Mode 1 is reduced for S-Ducts $3 \mathrm{~A}$ and 3B and for both geometries the distributions are broadly independent of Mach number. The spatial distributions of modes 0 to 5 are shown in Fig.12 and Fig. 13. Mode 0 is representative of the time-averaged total pressure distribution and modes 1 , 2 and 3 show distinct spatial mode distributions. Mode 1 has an anti-symmetric arrangement indicative of the underlying streamwise vortices associated with the secondary flows. As the POD coefficients are anti-symmetric it supports the evidence from the cross-correlation analysis at these locations which indicates an out of phase 
oscillation of the main flow features in this area. This is further supported by considering a frequency analysis of the POD coefficients for mode 1 (Fig. 14) which shows that the primary Strouhal number associated with mode 1 is around 0.4 (frequency $\approx 260 \mathrm{~Hz}$ ). The banded frequency spectrum for S-Duct $2 \mathrm{~A}$ also shows that the total pressure unsteadiness is dominated in this region of the mode 1 maxima within the Strouhal range 0.29-0.43 (Fig. 9). Mode 2 is shown to have a symmetric distribution with the local maximum located in relatively central position. A frequency analysis of the POD coefficient for mode 2, shows a clear peak at around $\mathrm{St}=0.75$ which also agrees with the spectrum peak within the Strouhal range 0.72-0.86 (Fig. 9). The assessment for S-Duct 2B when the Mach number reduces to 0.18 shows the mode shapes are relatively insensitive to the effect of Mach number with very little difference noted in modes 0-2 and minor differences observed in modes 3-5 (Fig. 12). For S-Duct 2A, by considering the spectrum of the POD coefficients, mode 1 is also noted as an asymmetric out of phase mode with a primary Strouhal number of around 0.4 and mode 2 is a symmetric unsteady mode within the duct centre at a Strouhal range 0.57-0.72. These are similar to the spectral distributions shown in shown in Fig. 9.

The POD mode distributions show distinct differences between S-Ducts 2 and S-Ducts 3. For S-Duct 3B the structures for modes 0-3 (Fig. 13) are generally similar to those for S-Ducts 2A and 2B (Fig. 12 ). There are some topological differences such as the clear radial movement in the centres of mode 1 as well as a shift in position for modes 2 and 3. For modes 4 and 5 the distributions are clearly different between those of S-Duct 2A, 2B and that shown for S-Duct 3B. For S-Duct 3A there are a few notable differences in comparison with S-Duct 3B and the effect of Mach number is not negligible. The geometry is the same for both of these configurations, but the AIP Mach number is 0.39 for $3 \mathrm{~A}$ and 0.18 for 3B. The first difference is in modes 1 and 2 where for $3 \mathrm{~B}$ these modes comprise an anti-symmetric shape and a single local maximum shape, respectively (Fig. 13). In comparison at the higher Mach number of 0.39 , these modes are reversed with the centre symmetric topology now more potent and comprising mode 1 .

Mode 3 and mode 5 are broadly the same for S-Duct 3A and 3B and there is a clear inversion of the distribution of local maxima and minima for mode 4. A frequency analysis of the POD mode coefficients showed that for SDuct $3 \mathrm{~A}$, mode 2 has a clear strong peak at about $\mathrm{St}=0.6$ although with is a relatively wide range of spectral content. Mode 1 had spectral peaks at about $\mathrm{St}=0.5$ and 0.7. Overall this is consistent with the distribution of pressure spectral analysis (Fig. 9) and indicates that the unsteadiness for S-Duct 3A is associated with a range of less distinct modes of a more amalgamated nature. This is because of the stronger secondary flows which results in the primary 
streamwise vortices migrating into a more central position. In addition, the centreline region is dominated by the classic diffusion separation which is associated with unsteady vorticity orthogonal to the r- $\theta$ plane and overall these two separated flows are more merged. This is notably different from the characteristics of S-Duct 2A in which these two flow features are more distinct in location and frequency. For S-Duct 3B the characteristics are slightly different again and the spectral analysis showed a clear single strong peak at about $\mathrm{St}=0.55$ which is associated with mode 1 although there are also other smaller local maxima at sub-harmonics of about 0.18 and 0.39 (Fig. 14). This indicates an out of phase lateral perturbation at a frequency of approximately $\mathrm{St}=0.55$ for S-Duct 3B. This postulated lateral oscillation was reported by Garnier ${ }^{11}$ at about $\mathrm{St}=0.48$, for the same geometry at similar flow conditions. There is also a secondary spectral peak for modes 2 and 3 at around $\mathrm{St}=0.85$.

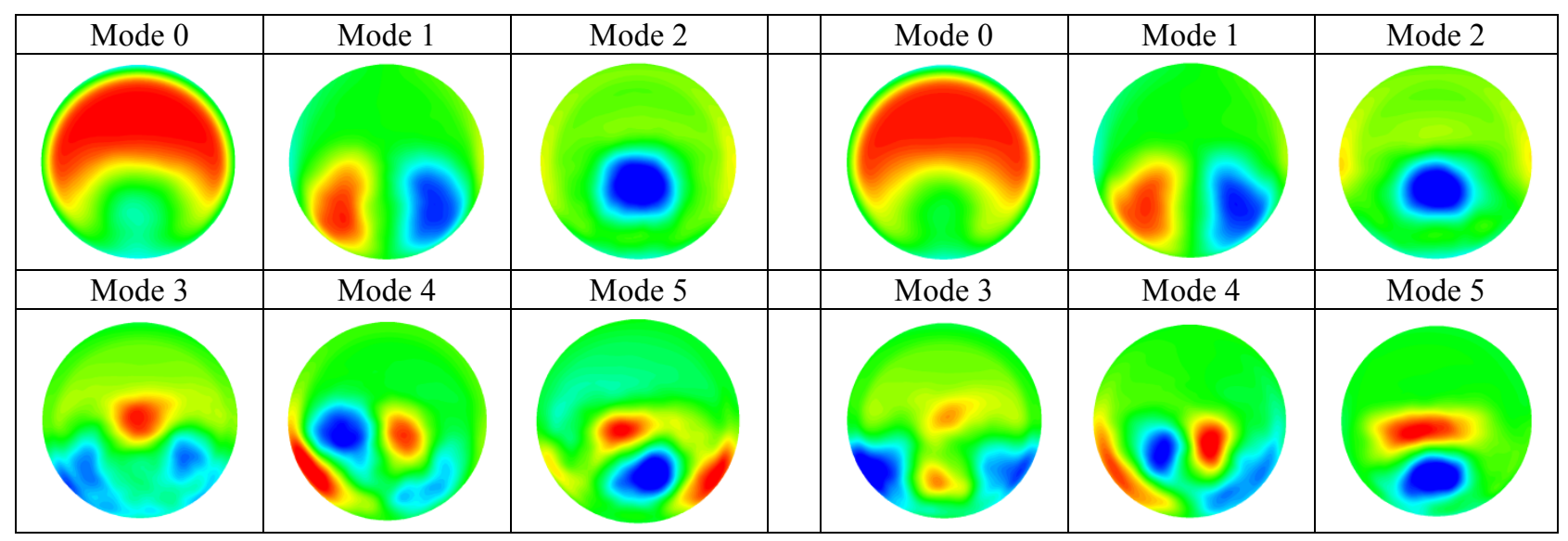

Fig. 12 AIP plane distribution of POD modes 0 to 5 for the cases S-Duct 2A (left) and 2B (right)

\begin{tabular}{|c|c|c|c|c|c|c|}
\hline Mode 0 & Mode 1 & Mode 2 & & Mode 0 & Mode 1 & Mode 2 \\
\hline & & & & & & \\
& & & & & & \\
\hline
\end{tabular}

Fig. 13 AIP plane distribution of POD modes 0 to 5 for the cases S-Duct 3A (left) and 3B (right) 
S-Duct 2B
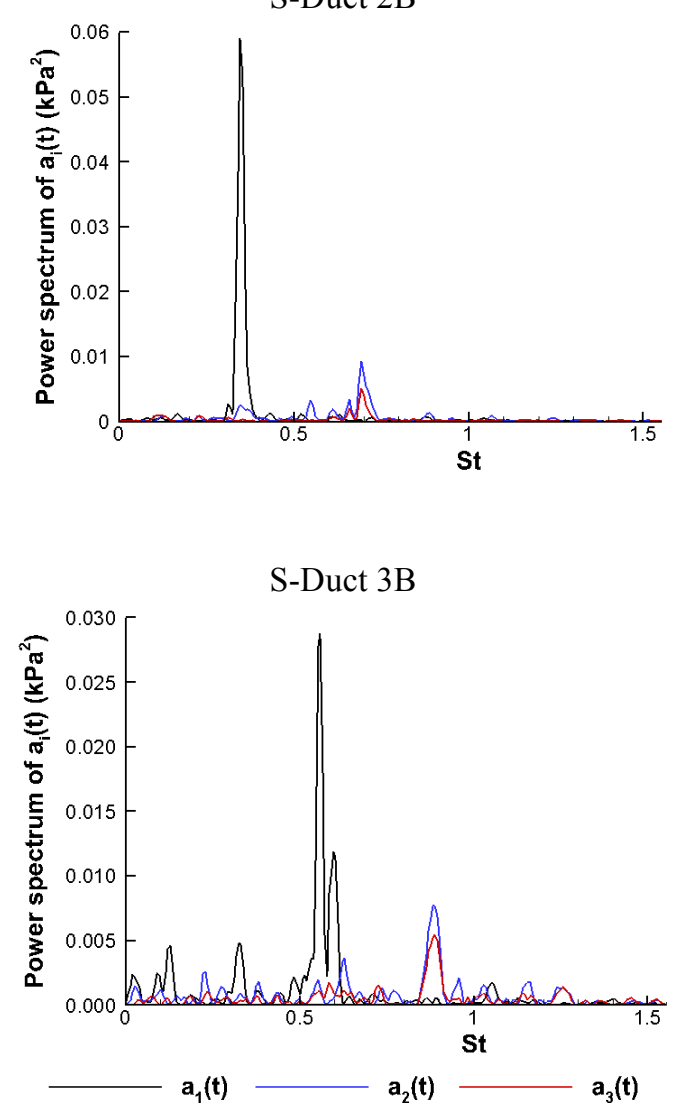

Fig. 14 Spectral distribution of the total pressure POD coefficients.

\section{Effect of POD modes on flow distortion}

Additional information on the relative importance of the POD modes for the dynamic aspects of the flow distortion metrics is of interest. This is particularly pertinent for the CDI-RDI metrics which exhibit notable levels of variation (Fig. 8). The impact of the individual POD modes was evaluated by reconstructing the AIP flow field snapshots for all the POD modes with the cumulative range of mode of interest omitted. This enabled the time history and statistics of the distortion metrics to be calculated for the time-series and the overall effect on the timeaveraged and distortion metric statistics to be quantified. Given the dynamic nature of the swirl distortion, the effect of the modes on the unsteady CDI and RDI metrics is a prime consideration. This is assessed by evaluating the unsteady reconstructed flow field based on the cumulative removal of the modes, as outlined in Fig. 15. The impact of the modes on the CDI standard deviation shows a generally monotonic reduction in the CDI standard deviation as more of the modes are removed. In general, S-Duct 2 is more affected by the lower modes than S-Duct 3 and for SDuct $2 \mathrm{~B}$, the unsteady CDI has reduced to about $50 \%$ when modes $1-10$ are removed. For S-Duct $2 \mathrm{~A}$, the reduction 
with the number of modes is not exactly monotonic which highlights and interaction between the different unsteady modes on the CDI, but the effect is modest. Overall it shows that for the unsteady elements of the CDI distortion, it is not strongly dominated by a single or small group of modes. The impact of modes 1 to 10 on the unsteady RDI highlights some differences between the ducts. S-Duct 2 and 3B show that mode 1 has a small effect and, although particular modes are more potent than others, it is not strongly dominated by an individual and that broadly the cumulative impact of the higher modes gradually accounts for the unsteadiness in RDI. S-Duct 3A, however, shows that mode 1 accounts for $30 \%$ of the unsteadiness and that modes $1-4$ account for about $55 \%$ of the unsteadiness. Apart from these elements, the modal reconstructions highlight that generally the unsteady distortion metrics are not strongly dominated by particular modes, but that the cumulative and interaction effects are important. Consequently, from the point of view of assessing the impact on engine compression systems, it indicates that a canonical approach to replicating the unsteady distortion elements, either computationally or experimentally, may not be appropriate.
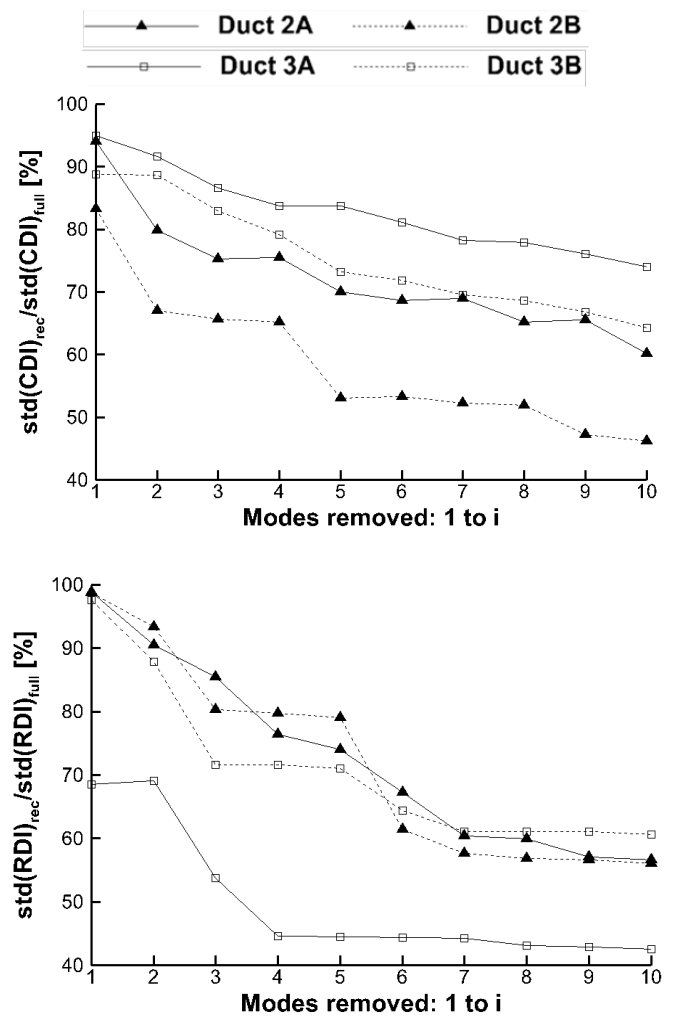

Fig. 15 Impact of POD modes on distortion metrics 


\section{Conclusion}

The unsteady flow field for a range of S-duct configurations has been simulated and assessed using a DDES computational method. The configurations encompass the effects of Mach number, Reynolds number and S-duct centreline offset. Analysis of the conventional distortion criteria highlights the main sensitivities to the S-duct configuration and quantifies the unsteady range of these parameters. These results illustrate the strongly dynamic nature of the flow field for both total pressure as well as swirl based distortion. For example the local swirl values are twice as large in the unsteady flow field in comparison with the time-averaged flow field. Statistical analysis of the distortion metrics shows that although the high-offset duct notably reduces the DC60 distortion the unsteady distortion of the RDI metric increases substantially. Analysis of the unsteady flow field for the low offset geometry, shows signature regions of unsteadiness which are postulated to be related to the classical secondary flows as well as to the streamwise flow separation. These signatures are changed by the higher offset geometry where the unsteadiness is more broadband and the distinction between these two mechanisms is less clear. For the low-offset geometry, an increase in Reynolds number at the same Mach number, generally provided a reduction in the timeaveraged distortion, but no notable reduction in the peak distortion metrics. For both the high- and low-offset ducts, an increase in Mach number reduces the duct pressure recovery, and there is a modest increase in the average $\mathrm{DC}(60)$ and $\mathrm{SC}(60)$ distortion metrics. A proper orthogonal decomposition of the total pressure field at the duct exit identifies the underpinning flow modes which are associated with the overall total pressure unsteadiness distributions. For the high-offset duct, the flow modes are notably different and highlight the reduced demarcation between the unsteady flow field mechanisms. For the low offset geometry, a spectral analysis of the time-history of the POD coefficients confirms the nature of the total pressure spectral distributions and identifies the associated POD modes. An initial assessment of the relative importance of the POD modes on the unsteady aspects of the distortion provided mixed results. The most notable indication is that the mode most associated with the centreline separation for the high-offset configuration has a substantial effect on the RDI distortion metric. Overall the work indicates the levels and sensitivities of total pressure and swirl based distortion metrics for a range of S-duct

configurations. Although the POD mode 0 dominates the time-averaged characteristics, the potent unsteady distortion metrics are found to be generally dependent on a much wider range of modes. 


\section{Acknowledgments}

The authors would like to thank Eric Garnier and Anne-Laure Delot from ONERA for providing images used in Fig. 4, Fig. 5, and Fig. 6.

\section{References}

${ }^{1}$ Cousins, W.T., "History, Philosophy, Physics, and Future Direction of Aircraft Propulsion System / Inlet Integration", Proceedings of ASME Turbo Expo 2004, Power for Land, Sea, and Air, Vol. 2, GT2004-54210, Vienna, Austria, June 2004, pp. 305-320.

Doi: $10.1115 / \mathrm{GT} 2004-54210$

${ }^{2}$ Society of Automotive Engineers, “A Methodology for Assessing Inlet Swirl Distortion,” SAE Aerospace Information Report 5686, Warrendale, PA, USA, 2007.

${ }^{3}$ Bissinger, N. C., and Breuer, T., "Basic Principles: Gas Turbine Compatibility - Intake Aerodynamic Aspects," Encyclopedia of Aerospace Engineering, John Wiley \& Sons, Ltd., , Dec. 2010.

Doi: 10.1002/9780470686652.eae487

${ }^{4}$ Mitchell, G. A., "Effect of Inlet Ingestion of a Wing Tip Vortex on Compressor Face Flow and Turbojet Stall Margin," Tech. Rep. TM X-3246, NASA, 1975.

${ }^{5}$ Mitchell, G. A., "Effect of Inlet Ingestion of a Wing Tip Vortex on Turbojet Stall Margin," NASA Tech. Rep. TM-X$71610,1974$.

${ }^{6}$ Meyer, W., Pazur, W., and Fottner, L., "The Influence of Intake Swirl Distortion on the Steady-State Performance of a Low Bypass Twin-Spool Engine", AGARD, Report CP-498, 1991.

${ }^{7}$ Bowditch, D. N., and Coltrin, R. E., “A Survey of Inlet/Engine Distortion Compatibility”, NASA TM-83421, 1983

${ }^{8}$ Wellborn, S. R., Okiishi, T. H., and Reichert, B.A., "A study of the Compressible Flow Through a Diffusing S-Duct," NASA TM-106411, 1993.

${ }^{9}$ Delot, A. L., and Scharnhorst, R. K., "A Comparison of Several CFD Codes with Experimental Data in a Diffusing SDuct," 49th AIAA/ASME/SAE/ASEE Joint Propulsion Conference, AIAA 2013-3796, San Jose, TX, USA, July 14-17, 2013.

Doi: $10.2514 / 6.2013-3796$

10 Fiola, C., and Agarwal, R. K., "Simulation of Secondary and Separated Flow in Diffusing S Ducts", AIAA Journal of Propulsion and Power, Vol. 31, No. 1, January-February 2015, pp. 180-191.

Doi: $10.2514 / 1 . B 35275$ 
${ }^{11}$ Garnier, E., "Flow Control by Pulsed Jet in a Curved S-Duct: A Spectral Analysis", AIAA Journal, Vol. 53, No. 10, Oct. 2015 , pp. 2813-2827.

Doi: $10.2514 / 1 . J 053422$

${ }^{12}$ Delot, A. L., Garnier, E., and Pagan, D., "Flow Control in a High-Offset Subsonic Air Intake," 47th AIAA/ASME/SAE/ASEE Joint Propulsion Conference \& Exhibit, AIAA 2011-5569, San Diego, CA, USA, 31 July - 03 August 2011.

Doi: $10.2514 / 6.2011-5569$

${ }^{13}$ Garnier, E., Leplat, M, Monnier, J. C., and Delva, J., "Flow control by pulsed jet in a highly bended S-duct," AIAA 20123250, 6th AIAA Flow Control Conference, NO , Louisiana, USA, 25-28 June 2012.

Doi: $10.2514 / 6.2012-3250$

${ }^{14}$ Berens, T. M., Delot, A. L., Chevalier, M., and Van Muijden, "Numerical Simulations for High Offset Intake Diffuser Flows," 52nd AIAA Aerospace Sciences Meeting - AIAA SciTech 2014, AIAA 2014-0371, National Harbor, Maryland, USA, 1317 January 2014.

Doi: $10.2514 / 6.2014-0371$

${ }^{15}$ Delot, A. L., Berens, T. M., Tormalm, M., Säterskog, M., and Ceresola, N., "DES Computations for a Subsonic UAV Configuration with a Highly Integrated S-Shaped Inlet Duct," 52nd AIAA Aerospace Sciences Meeting - AIAA SciTech 2014, AIAA 2014-0723, National Harbor, Maryland, USA, 13-17 January 2014.

Doi: $10.2514 / 6.2014-0723$

${ }^{16}$ Deck, S., "Recent Improvements of the Zonal Detached Eddy Simulation (ZDES) formulation", Theoretical and Computational Fluid Dynamics, Vol., 26, 2012, pp. 523-550

Doi:10.1007/s00162-011-0240-z

${ }^{17}$ Harloff, G., Reichert B. A., and Wellborn S. R., "Navier-Stokes Analysis and Experimental Data Comparison of Compressible Flow in a Diffusing S-Duct”, NASA TM-105683, February 1992.

Doi: $10.2514 / 6.1992-2699$

${ }^{18}$ Society of Automotive Engineers, "Inlet Total-Pressure-Distortion Considerations for Gas-Turbine Engines," SAE Aerospace Information Report 1419a, Warrendale, PA, USA, 1999.

${ }^{19}$ Seddon, J. , and Goldsmith, E.L., Intake Aerodynamics, Second Edition, Blackwell Science Ltd, 1999, Chap. 11, pp. 281284.

${ }^{20}$ Cousins, W. T., “The Dynamics of Stall and Surge Behaviour in Axial-Centrifugal Compressors," Ph.D. Dissertation, Virginia Polytechnic Institute and State University, 1997. 
${ }^{21}$ Zantopp, S., MacManus, D., and Murphy, J., “Computational and experimental study of intake ground vortices”, The Aeronautical Journal, 114, 2010, pp 769-784.

Doi:10.1017/S0001924000004255

${ }^{22}$ Spalart, P. S., “Detached-Eddy Simulation,” Annual Review of Fluid Mechanics, Vol. 41, Aug. 2008, pp. 181-202.

Doi: 10.1146/annurev.fluid.010908.165130.

${ }^{23}$ Chevalier, M., and Peng, S.-H., "Detached Eddy Simulation of Turbulent Flow in a Highly Offset Intake Diffuser", Progress in Hybrid RANS-LES Modelling, Notes on Numerical Fluid Mechanics and Multidisciplinary Design, Vol. 111, Springer-Verlag Berlin, 2010, pp. 111-121.

${ }^{24}$ Spalart, P., Deck, S., Shur, K., Squires, M., Strelets, A., Travin, A., “A New Version of Detached Eddy Simulation, Resistant to Ambiguous Grid Densities", Theoretical and Computational Fluid Dynamics, Vol., 20, 2006, pp. $181-195$. doi:10.1007/s00162-006-0015-0

${ }^{25}$ ANSYS, “ANSYS FLUENT Theory Guide”, ANSYS, Inc, Southpointe, 275 Technology Drive, Canonsburg, PA, 15317. Release 14.0. November 2011. Chap. 20, pp. 641-675

${ }^{26}$ ANSYS, “ANSYS FLUENT User Guide”, ANSYS, Inc, Southpointe, 275 Technology Drive, Canonsburg, PA, 15317. Release 14.0. November 2011. Chap. 29, pp 1321-1323, 1365-1374

${ }^{27}$ Georgiadis , N. J., Rizzetta , D. P., and Fureby, C., "Large-Eddy Simulation: Current Capabilities, Recommended Practices, and Future Research”, AIAA Journal, Vol. 48, No. 8, Aug. 2010.

Doi: $10.2514 / 1 . J 050232$

${ }^{28}$ Roache, P. J., "Perspective: a Method for Uniform Reporting of Grid Refinement Study", Journal of Fluid Engineering, Vol. 116, No 3, 1994, pp. 405413.

Doi:10.1115/1.2910291

${ }^{29}$ Kostas, J., Soria, J., and Chong, M. S., “A Comparison Between Snapshot POD Analysis of PIV Velocity and Vorticity Data," Experiments in Fluids, Vol. 38, No. 2, 2005, pp 146-160.

Doi: $10.1007 / \mathrm{s} 00348-004-0873-$

${ }^{30}$ Kostas, J., Soria, J., and Chong, M. S., "Particle Image Velocimetry Measurements of a Backward-Facing Step Flow," Experiments in Fluids, Vol. 33, No. 6, 2002, pp 838-853.

Doi: 10.1007/s00348-002-0521-9

${ }^{31}$ Chen, H., Reuss, D. L., Hung, D. L., and Sick, V., “A practical guide for using proper orthogonal decomposition in engine research,” International Journal of Engine Research, Vol. 14, No. 4, 2013, pp 307-319.

Doi: $10.1177 / 1468087412455748$ 
${ }^{32}$ Gurka, R., Liberzon, A., and Hetsroni, G., "POD of vorticity fields: A method for spatial characterisation of coherent structures, ", International Journal of Heat and Fluid Flow, Vol. 27, No. 3, 2006, pp 416-423.

Doi:10.1016/j.ij heatfluidflow.2006.01.001

${ }^{33}$ Kalpakli Vester, A., Orlü, R., and Alfredsson, P. H., "POD analysis of the turbulent flow downstream a mild and sharp bend”, Experiments in Fluids, Vol. 56, No. 3, Article id. 57, March 2005.

Doi: $10.1007 / \mathrm{s} 00348-015-1926-6$

${ }^{34}$ Holmes, P., Lumley, J. L., Berzook, G., and Rowley, C. W., Turbulence, Coherent Structures, Dynamical Systems and Symmetry, $2^{\text {nd }}$ edition, Cambridge University Press, Cambridge, England, UK, 2012, Chap. 3.

${ }^{35}$ Lumley, J. L., Stochastic Tools in turbulence, Dover Publications, Inc., New York, USA, 2007, Chap. 3, pp. 57-59.

${ }^{36}$ Berzook, G., Holmes, P., and Lumley, J. L., "The proper orthogonal decomposition in the analysis of turbulent flows", Annual Review of Fluid Mechanics, Vol. 25, 1993, pp 539-75.

Doi: 10.1146/annurev.fl.25.010193.002543

${ }^{37}$ Sirovich, L., "Turbulence and the dynamics of coherent structures. Part 1: Coherent structures," Quarterly of Applied Mathematics, Vol. XLV, Oct. 1987, pp 561-571. 
2017-03-29

\section{Complex aero-engine intake ducts and dynamic distortion}

MacManus, David G.

American Institute of Aeronautics and Astronautics

MacManus DG, Chiereghin N, Gil Prieto D, Zachos P. (2017) Complex aeroengine intake ducts and dynamic distortion. AIAA Journal, Volume 55, Issue 7, 2017, pp. 2395-2409

http://dx.doi.org/10.2514/1.J054905

Downloaded from Cranfield Library Services E-Repository 